\title{
Calculation of Atomic Data and Gain Coefficient for XUV \& Soft X-Ray Laser Emission from Ge XXIII
}

\author{
Wessameldin S. Abdelaziz \\ National Institute of Laser Enhanced Sciences, Cairo University, Giza, Egypt \\ Email: wessamlaser@yahoo.com
}

Received 21 June 2014; revised 18 July 2014; accepted 12 August 2014

Copyright (C) 2014 by author and Scientific Research Publishing Inc.

This work is licensed under the Creative Commons Attribution International License (CC BY).

http://creativecommons.org/licenses/by/4.0/

(c) () Open Access

\begin{abstract}
Energy levels, transition probabilities, oscillator strengths, and collision strengths have been calculated for transitions in Ne-like Ge. The data refer to a 241 fine-structure levels belonging to the configurations $1 s^{2} 2 s^{2} 2 p^{5} n l, 1 s^{2} 2 s^{1} 2 p^{6} n l(n=3,4,5,6 ; 1=s, p, d, f, g$ and $h)$, which have been calculated by the fully relativistic flexible atomic code (FAC). These data are used in the determination of the reduced population for the $\mathbf{2 4 1}$ fine structure levels and gain coefficients over a wide range of electron densities (from $2 \times 10^{+20}$ to $4 \times 10^{+22} \mathrm{~cm}^{-3}$ ) and at various electron plasma temperatures $(650,850,1050,1250,1450,1650,1850) \mathrm{eV}$ by using the MATLAB R2013a Computer program for solving simultaneous coupled rate equations. The reduced population for the 241 fine structure levels the gain coefficients for those transitions with positive population inversion factor are determined and plotted against the electron density.
\end{abstract}

\section{Keywords}

Energy Levels, Transition Probabilities, Oscillator Strengths, Collision Strengths

\section{Introduction}

The concept for x-ray lasers went back to the 1965s, when it was based on the laser-produced plasmas by electron de-excitation having a promising interest science, which was first proposed by the Soviet scientists Gudzenko and Shelepin [1]. They thought that the short wavelength laser in the x-ray region of the electromagnetic spectrum needed a large energy gap which was sustained in the highly ionized ions. After ionization, in the equilibrium plasma, ions having specific number of electrons such as 2 (helium-like), 10 (neon-like), 28 (nickel-like) and 46 (palladium-like) were relatively stable and survived in a wide range of temperature and density [2] [3]. X-ray 
lasers pumping methods are electron collisional excitation, photo excitation, charge transfer, electron collisional recombination and de-electronic recombination pumping using a picosecond chirped pulse amplification (CPA) pulse [4], a capillary discharge [5] [6] and a free electron laser. However, the electron collisional excitation pumping of the inner shell and outer shell of highly ionization states have shown a more stable and a higher output [7] [8]. Thus, the electron collisional pumping proved to be the most efficient method in producing the x-ray lasers.

Experimentally there exist in the literature some studies trying to develop high-efficiency X-ray laser with significant gain, for example, [3] [9] proposed the original mechanism for demonstrating X-ray lasing by resonant photo pumping. Several authors during the past three decades [10] [11] have studied this lasing mechanism experimentally and theoretically, in the hope of developing high-efficiency X-ray laser. In another study by Qi, N. and Krishnan, M. [12], the shortest wavelength at which the significant gain had been measured using the resonant photo pumping was in the beryllium-like carbon at $2163 \AA$, which was far from the X-ray spectral region.

In this paper, we calculate energy levels for 241 fine-structure states using a fully relativistic approach based on Dirac equation (see Table 1). Weighted Oscillator strengths, spontaneous radiative decay rates are calculated in the single multipole approximation (see Table 2), and Collision strengths by electron impact using the factorization-interpolation method are calculated in the distorted wave approximation. Effective collision strengths are calculated by interpolating the data from the collision strengths and integrating over Max wellian distribution at different temperatures. Rate coefficients are calculated from effective collision strengths using a formula that will be described later in this paper. Then, we predict the reduced population and gain coefficient for Ge XXIII by a steady state equation in the collisional radiative model after achieving a population inversion between the allowed transition states.

Table 1. Energy levels of Ge XXIII, and their threshold energies (in eV).

\begin{tabular}{|c|c|c|c|c|c|c|c|c|c|}
\hline Index & $\begin{array}{c}\text { State } \\
\text { Configuration }\end{array}$ & $\mathrm{J}^{\pi}$ & FAC & COWAN & NIST & Exp. & CIV3 & $\mathrm{MCDF}$ & $\mathrm{RCI}$ \\
\hline 1 & $2 p^{6}$ & $0^{\mathrm{e}}$ & $0.00000 \mathrm{E}+00$ & $0.00000 \mathrm{E}+00$ & $0.00000 \mathrm{E}+00$ & $0.00000 \mathrm{E}+00$ & $0.00000 \mathrm{E}+00$ & $0.00000 \mathrm{E}+00$ & $0.00000 \mathrm{E}+00$ \\
\hline 2 & $2 \mathrm{p}_{312} 3 \mathrm{~s}_{112}$ & $2^{\circ}$ & $1.23421 \mathrm{E}+03$ & $1.23585 \mathrm{E}+03$ & & $1.236122 \mathrm{E}+03$ & $1.23596 \mathrm{E}+03$ & $1.234804 \mathrm{E}+03$ & $1.236931 \mathrm{E}+03$ \\
\hline 3 & $2 \mathrm{p}_{312} 3 \mathrm{~s}_{112}$ & $1^{\circ}$ & $1.23709 \mathrm{E}+03$ & $1.23863 \mathrm{E}+03$ & $1.23900 \mathrm{E}+03$ & $1.238602 \mathrm{E}+03$ & $1.23859 \mathrm{E}+03$ & $1.237659 \mathrm{E}+03$ & $1.239498 \mathrm{E}+03$ \\
\hline 4 & $2 \mathrm{p}_{112} 3 \mathrm{~s}_{112}$ & $0^{\circ}$ & $1.26664 \mathrm{E}+03$ & $1.26835 \mathrm{E}+03$ & & $1.268358 \mathrm{E}+03$ & $1.26828 \mathrm{E}+03$ & $1.267259 \mathrm{E}+03$ & $1.269494 \mathrm{E}+03$ \\
\hline 5 & $2 \mathrm{p}_{112} 3 \mathrm{~s}_{112}$ & $1^{\circ}$ & $1.26822 \mathrm{E}+03$ & $1.26994 \mathrm{E}+03$ & $1.27009 \mathrm{E}+03$ & $1.269598 \mathrm{E}+03$ & $1.26978 \mathrm{E}+03$ & $1.268864 \mathrm{E}+03$ & $1.270625 \mathrm{E}+03$ \\
\hline 6 & $2 \mathrm{p}_{312} 3 \mathrm{p}_{112}$ & $1^{\mathrm{e}}$ & $1.27690 \mathrm{E}+03$ & $1.27848 \mathrm{E}+03$ & --- & $1.278277 \mathrm{E}+03$ & $1.27857 \mathrm{E}+03$ & $1.277620 \mathrm{E}+03$ & $1.280475 \mathrm{E}+03$ \\
\hline 7 & $2 \mathrm{p}_{312} 3 \mathrm{p}_{1 / 2}$ & $2^{\mathrm{e}}$ & $1.28046 \mathrm{E}+03$ & $1.28213 \mathrm{E}+03$ & --- & $1.281997 \mathrm{E}+03$ & $1.28213 \mathrm{E}+03$ & $1.280943 \mathrm{E}+03$ & $1.283104 \mathrm{E}+03$ \\
\hline 8 & $2 \mathrm{p}_{312} 3 \mathrm{p}_{312}$ & $3^{\mathrm{e}}$ & $1.28642 \mathrm{E}+03$ & $1.28801 \mathrm{E}+03$ & --- & $1.288196 \mathrm{E}+03$ & $1.28801 \mathrm{E}+03$ & $1.286986 \mathrm{E}+03$ & $1.288182 \mathrm{E}+03$ \\
\hline 9 & $2 \mathrm{p}_{312} 3 \mathrm{p}_{312}$ & $1^{\mathrm{e}}$ & $1.28726 \mathrm{E}+03$ & $1.28859 \mathrm{E}+03$ & --- & $1.288196 \mathrm{E}+03$ & $1.28869 \mathrm{E}+03$ & $1.287800 \mathrm{E}+03$ & $1.288267 \mathrm{E}+03$ \\
\hline 10 & $2 \mathrm{p}_{312} 3 \mathrm{p}_{312}$ & $2^{\mathrm{e}}$ & $1.29059 \mathrm{E}+03$ & $1.29201 \mathrm{E}+03$ & $1.29204 \mathrm{E}+03$ & $1.291915 \mathrm{E}+03$ & $1.29190 \mathrm{E}+03$ & $1.291035 \mathrm{E}+03$ & $1.291087 \mathrm{E}+03$ \\
\hline 11 & $2 \mathrm{p}_{312} 3 \mathrm{p}_{312}$ & $0^{\mathrm{e}}$ & $1.30216 \mathrm{E}+03$ & $1.30328 \mathrm{E}+03$ & --- & $1.303074 \mathrm{E}+03$ & $1.30354 \mathrm{E}+03$ & $1.302522 \mathrm{E}+03$ & $1.301413 \mathrm{E}+03$ \\
\hline 12 & $2 \mathrm{p}_{1,2} 3 \mathrm{p}_{1 \mathrm{1}}$ & $1^{\mathrm{e}}$ & $1.31186 \mathrm{E}+03$ & $1.31352 \mathrm{E}+03$ & --- & $1.314232 \mathrm{E}+03$ & $1.31355 \mathrm{E}+03$ & $1.312405 \mathrm{E}+03$ & $1.314407 \mathrm{E}+03$ \\
\hline 13 & $2 \mathrm{p}_{1,2} 3 \mathrm{p}_{312}$ & $1^{\mathrm{e}}$ & $1.31985 \mathrm{E}+03$ & $1.32137 \mathrm{E}+03$ & --- & $1.321671 \mathrm{E}+03$ & $1.32122 \mathrm{E}+03$ & $1.320429 \mathrm{E}+03$ & $1.322501 \mathrm{E}+03$ \\
\hline 14 & $2 \mathrm{p}_{1,2} 3 \mathrm{p}_{312}$ & $2^{\mathrm{e}}$ & $1.32083 \mathrm{E}+03$ & $1.32231 \mathrm{E}+03$ & $1.32254 \mathrm{E}+03$ & $1.322911 \mathrm{E}+03$ & $1.32229 \mathrm{E}+03$ & $1.321340 \mathrm{E}+03$ & $1.323593 \mathrm{E}+03$ \\
\hline 15 & $2 \mathrm{p}_{1,2} 3 \mathrm{p}_{112}$ & $0^{\mathrm{e}}$ & $1.33447 \mathrm{E}+03$ & $1.33333 \mathrm{E}+03$ & $1.33333 \mathrm{E}+03$ & $1.332830 \mathrm{E}+03$ & $1.33321 \mathrm{E}+03$ & $1.332304 \mathrm{E}+03$ & $1.334537 \mathrm{E}+03$ \\
\hline 16 & $2 \mathrm{p}_{312} 3 \mathrm{~d}_{312}$ & $0^{\circ}$ & $1.34070 \mathrm{E}+03$ & $1.34234 \mathrm{E}+03$ & -- & $1.342749 \mathrm{E}+03$ & $1.34218 \mathrm{E}+03$ & $1.341534 \mathrm{E}+03$ & $1.343407 \mathrm{E}+03$ \\
\hline 17 & $2 \mathrm{p}_{312} 3 \mathrm{~d}_{312}$ & $1^{\mathrm{o}}$ & $1.34247 \mathrm{E}+03$ & $1.34422 \mathrm{E}+03$ & $1.34473 \mathrm{E}+03$ & $1.343989 \mathrm{E}+03$ & $1.34403 \mathrm{E}+03$ & $1.343296 \mathrm{E}+03$ & $1.345574 \mathrm{E}+03$ \\
\hline 18 & $2 \mathrm{p}_{312} 3 \mathrm{~d}_{512}$ & $4^{\circ}$ & $1.34553 \mathrm{E}+03$ & $1.34748 \mathrm{E}+03$ & --- & --- & $1.34724 \mathrm{E}+03$ & $1.346399 \mathrm{E}+03$ & $1.348306 \mathrm{E}+03$ \\
\hline 19 & $2 \mathrm{p}_{312} 3 \mathrm{~d}_{5 \backslash 2}$ & $2^{\circ}$ & $1.34575 \mathrm{E}+03$ & $1.34753 \mathrm{E}+03$ & --- & $1.347708 \mathrm{E}+03$ & $1.34730 \mathrm{E}+03$ & $1.346560 \mathrm{E}+03$ & $1.348784 \mathrm{E}+03$ \\
\hline 20 & $2 \mathrm{p}_{312} 3 \mathrm{~d}_{312}$ & $3^{\circ}$ & $1.34594 \mathrm{E}+03$ & $1.34772 \mathrm{E}+03$ & --- & $1.347708 \mathrm{E}+03$ & $1.34779 \mathrm{E}+03$ & $1.346843 \mathrm{E}+03$ & $1.348794 \mathrm{E}+03$ \\
\hline 21 & $2 \mathrm{p}_{312} 3 \mathrm{~d}_{312}$ & $2^{\circ}$ & $1.34871 \mathrm{E}+03$ & $1.35031 \mathrm{E}+03$ & --- & $1.350188 \mathrm{E}+03$ & $1.35025 \mathrm{E}+03$ & $1.349561 \mathrm{E}+03$ & $1.351217 \mathrm{E}+03$ \\
\hline
\end{tabular}




\section{Continued}

\begin{tabular}{|c|c|c|c|c|c|c|c|c|c|}
\hline 22 & $2 \mathrm{p}_{312} 3 \mathrm{~d}_{512}$ & $3^{\circ}$ & $1.35077 \mathrm{E}+03$ & $1.35267 \mathrm{E}+03$ & --- & $1.352668 \mathrm{E}+03$ & $1.35230 \mathrm{E}+03$ & $1.351588 \mathrm{E}+03$ & $1.353658 \mathrm{E}+03$ \\
\hline 23 & $2 \mathrm{p}_{312} 3 \mathrm{~d}_{512}$ & $1^{\circ}$ & $1.35938 \mathrm{E}+03$ & $1.35991 \mathrm{E}+03$ & $1.36085 \mathrm{E}+03$ & $1.361346 \mathrm{E}+03$ & $1.36075 \mathrm{E}+03$ & $1.360284 \mathrm{E}+03$ & $1.362151 \mathrm{E}+03$ \\
\hline 24 & $2 \mathrm{p}_{112} 3 \mathrm{~d}_{312}$ & $2^{\circ}$ & $1.37811 \mathrm{E}+03$ & $1.37920 \mathrm{E}+03$ & --- & --- & $1.37955 \mathrm{E}+03$ & $1.378922 \mathrm{E}+03$ & $1.380718 \mathrm{E}+03$ \\
\hline 25 & $2 \mathrm{p}_{112} 3 \mathrm{~d}_{512}$ & $2^{\circ}$ & $1.37971 \mathrm{E}+03$ & $1.38104 \mathrm{E}+03$ & --- & --- & $1.38101 \mathrm{E}+03$ & $1.380575 \mathrm{E}+03$ & $1.382465 \mathrm{E}+03$ \\
\hline 26 & $2 \mathrm{p}_{112} 3 \mathrm{~d}_{512}$ & $3^{\circ}$ & $1.38086 \mathrm{E}+03$ & $1.38195 \mathrm{E}+03$ & --- & $1.383664 \mathrm{E}+03$ & $1.38231 \mathrm{E}+03$ & $1.381764 \mathrm{E}+03$ & $1.383652 \mathrm{E}+03$ \\
\hline 27 & $2 \mathrm{p}_{112} 3 \mathrm{~d}_{312}$ & $1^{\circ}$ & $1.38931 \mathrm{E}+03$ & $1.38884 \mathrm{E}+03$ & $1.38825 \mathrm{E}+03$ & $1.391103 \mathrm{E}+03$ & $1.39058 \mathrm{E}+03$ & $1.389940 \mathrm{E}+03$ & $1.392453 \mathrm{E}+03$ \\
\hline 28 & $2 \mathrm{~s}_{112} 3 \mathrm{~s}_{122}$ & $1^{\mathrm{e}}$ & $1.42808 \mathrm{E}+03$ & --- & --- & --- & $1.40081 \mathrm{E}+03$ & --- & $1.430234 \mathrm{E}+03$ \\
\hline 29 & $2 \mathrm{~s}_{112} 3 \mathrm{~s}_{121}$ & $0^{\mathrm{e}}$ & $1.43744 \mathrm{E}+03$ & --- & --- & --- & $1.40856 \mathrm{E}+03$ & --- & $1.438962 \mathrm{E}+03$ \\
\hline 30 & $2 \mathrm{~s}_{112} 3 \mathrm{p}_{112}$ & $0^{\circ}$ & $1.47300 \mathrm{E}+03$ & --- & --- & --- & $1.43866 \mathrm{E}+03$ & --- & $1.474996 \mathrm{E}+03$ \\
\hline 31 & $2 \mathrm{~s}_{112} 3 \mathrm{p}_{112}$ & $1^{\circ}$ & $1.47381 \mathrm{E}+03$ & --- & $1.47132 \mathrm{E}+03$ & --- & $1.43962 \mathrm{E}+03$ & --- & $1.476085 \mathrm{E}+03$ \\
\hline 32 & $2 s_{112} 3 p_{312}$ & $2^{\circ}$ & $1.48066 \mathrm{E}+03$ & --- & --- & --- & $1.44646 \mathrm{E}+03$ & --- & $1.482977 \mathrm{E}+03$ \\
\hline 33 & $2 \mathrm{~s}_{112} 3 \mathrm{p}_{312}$ & $1^{\circ}$ & $1.48318 \mathrm{E}+03$ & --- & $1.48074 \mathrm{E}+03$ & --- & $1.44867 \mathrm{E}+03$ & --- & $1.485577 \mathrm{E}+03$ \\
\hline 34 & $2 \mathrm{~s}_{112} 3 \mathrm{~d}_{312}$ & $1^{\mathrm{e}}$ & $1.53678 \mathrm{E}+03$ & --- & --- & --- & $1.49950 \mathrm{E}+03$ & --- & $1.539105 \mathrm{E}+03$ \\
\hline 35 & $2 \mathrm{~s}_{112} 3 \mathrm{~d}_{312}$ & $2^{\mathrm{e}}$ & $1.53722 \mathrm{E}+03$ & --- & --- & --- & $1.50003 \mathrm{E}+03$ & --- & $1.539522 \mathrm{E}+03$ \\
\hline 36 & $2 \mathrm{~s}_{112} 3 \mathrm{~d}_{512}$ & $3^{\mathrm{e}}$ & $1.53813 \mathrm{E}+03$ & --- & --- & --- & $1.50093 \mathrm{E}+03$ & --- & $1.540470 \mathrm{E}+03$ \\
\hline 37 & $2 \mathrm{~s}_{112} 3 \mathrm{~d}_{512}$ & $2^{\mathrm{e}}$ & $1.54524 \mathrm{E}+03$ & --- & --- & --- & $1.50787 \mathrm{E}+03$ & --- & $1.547695 \mathrm{E}+03$ \\
\hline 38 & $2 \mathrm{p}_{312} 4 \mathrm{~s}_{112}$ & $2^{\circ}$ & $1.67001 \mathrm{E}+03$ & $1.67259 \mathrm{E}+03$ & --- & --- & --- & --- & -- \\
\hline 39 & $2 \mathrm{p}_{312} 4 \mathrm{~s}_{112}$ & $1^{\circ}$ & $1.67092 \mathrm{E}+03$ & $1.67259 \mathrm{E}+03$ & $1.67250 \mathrm{E}+03$ & --- & --- & --- & --- \\
\hline 40 & $2 p_{312} 4 p_{112}$ & $1^{\mathrm{e}}$ & $1.68781 \mathrm{E}+03$ & $1.69062 \mathrm{E}+03$ & --- & --- & --- & --- & --- \\
\hline 41 & $2 p_{312} 4 p_{112}$ & $2^{\mathrm{e}}$ & $1.68868 \mathrm{E}+03$ & $1.69125 \mathrm{E}+03$ & --- & --- & --- & --- & --- \\
\hline 42 & $2 \mathrm{p}_{312} 4 \mathrm{p}_{312}$ & $3^{\mathrm{e}}$ & $1.69117 \mathrm{E}+03$ & $1.69380 \mathrm{E}+03$ & -- & -- & --- & --- & --- \\
\hline 43 & $2 \mathrm{p}_{312} 4 \mathrm{p}_{312}$ & $1^{\mathrm{e}}$ & $1.69150 \mathrm{E}+03$ & $1.69405 \mathrm{E}+03$ & --- & --- & --- & --- & --- \\
\hline 44 & $2 p_{312} 4 p_{312}$ & $2^{\mathrm{e}}$ & $1.69258 \mathrm{E}+03$ & $1.69497 \mathrm{E}+03$ & --- & --- & --- & --- & --- \\
\hline 45 & $2 p_{312} 4 p_{312}$ & $0^{\mathrm{e}}$ & $1.69841 \mathrm{E}+03$ & $1.69999 \mathrm{E}+03$ & --- & --- & --- & --- & --- \\
\hline 46 & $2 \mathrm{p}_{112} 4 \mathrm{~s}_{112}$ & $0^{\circ}$ & $1.70254 \mathrm{E}+03$ & $1.70497 \mathrm{E}+03$ & --- & --- & --- & --- & --- \\
\hline 47 & $2 \mathrm{p}_{112} 4 \mathrm{~s}_{112}$ & $1^{\circ}$ & $1.70295 \mathrm{E}+03$ & $1.70497 \mathrm{E}+03$ & $1.70466 \mathrm{E}+03$ & -- & --- & -- & -- \\
\hline 48 & $2 \mathrm{p}_{312} 4 \mathrm{~d}_{312}$ & $0^{\circ}$ & $1.71168 \mathrm{E}+03$ & $1.71460 \mathrm{E}+03$ & --- & --- & --- & --- & --- \\
\hline 49 & $2 \mathrm{p}_{312} 4 \mathrm{~d}_{312}$ & $1^{\circ}$ & $1.71237 \mathrm{E}+03$ & $1.71521 \mathrm{E}+03$ & --- & --- & --- & --- & --- \\
\hline 50 & $2 \mathrm{p}_{312} 4 \mathrm{~d}_{512}$ & $4^{\circ}$ & $1.71336 \mathrm{E}+03$ & $1.71608 \mathrm{E}+03$ & --- & --- & --- & -- & -- \\
\hline 51 & $2 \mathrm{p}_{312} 4 \mathrm{~d}_{312}$ & $3^{\circ}$ & $1.71340 \mathrm{E}+03$ & $1.71621 \mathrm{E}+03$ & -- & -- & --- & -- & -- \\
\hline 52 & $2 \mathrm{p}_{312} 4 \mathrm{~d}_{512}$ & $2^{\circ}$ & $1.71356 \mathrm{E}+03$ & $1.71631 \mathrm{E}+03$ & --- & --- & --- & -- & --- \\
\hline 53 & $2 \mathrm{p}_{312} 4 \mathrm{~d}_{312}$ & $2^{\circ}$ & $1.71440 \mathrm{E}+03$ & $1.71704 \mathrm{E}+03$ & --- & -- & --- & -- & --- \\
\hline 54 & $2 \mathrm{p}_{312} 4 \mathrm{~d}_{512}$ & $3^{\circ}$ & $1.71516 \mathrm{E}+03$ & $1.71772 \mathrm{E}+03$ & -- & -- & --- & -- & --- \\
\hline 55 & $2 \mathrm{p}_{312} 4 \mathrm{~d}_{512}$ & $1^{\circ}$ & $1.71893 \mathrm{E}+03$ & $1.72055 \mathrm{E}+03$ & $1.72078 \mathrm{E}+05$ & --- & --- & --- & --- \\
\hline 56 & $2 \mathrm{p}_{112} 4 \mathrm{p}_{112}$ & $1^{\mathrm{e}}$ & $1.72080 \mathrm{E}+03$ & $1.72379 \mathrm{E}+03$ & --- & --- & --- & -- & --- \\
\hline 57 & $2 p_{112} 4 p_{312}$ & $1^{\mathrm{e}}$ & $1.72399 \mathrm{E}+03$ & $1.72689 \mathrm{E}+03$ & --- & --- & --- & -- & --- \\
\hline 58 & $2 \mathrm{p}_{12} 4 \mathrm{p}_{312}$ & $2^{\mathrm{e}}$ & $1.72433 \mathrm{E}+03$ & $1.72708 \mathrm{E}+03$ & --- & -- & --- & -- & --- \\
\hline 59 & $2 \mathrm{p}_{312} 4 \mathrm{f}_{512}$ & $1^{\mathrm{e}}$ & $1.72480 \mathrm{E}+03$ & $1.72713 \mathrm{E}+03$ & -- & --- & --- & -- & -- \\
\hline 60 & $2 \mathrm{p}_{312} 4 \mathrm{f}_{512}$ & $4^{\mathrm{e}}$ & $1.72501 \mathrm{E}+03$ & $1.72742 \mathrm{E}+03$ & -- & -- & --- & -- & -- \\
\hline 61 & $2 \mathrm{p}_{312} 4 \mathrm{f}_{712}$ & $2^{\mathrm{e}}$ & $1.72515 \mathrm{E}+03$ & $1.72746 \mathrm{E}+03$ & --- & --- & --- & --- & --- \\
\hline
\end{tabular}




\section{Continued}

\begin{tabular}{|c|c|c|c|c|c|c|c|c|c|}
\hline 62 & $2 \mathrm{p}_{312} 4 \mathrm{f}_{712}$ & $5^{\mathrm{e}}$ & $1.72516 \mathrm{E}+03$ & $1.72764 \mathrm{E}+03$ & --- & --- & --- & --- & --- \\
\hline 63 & $2 \mathrm{p}_{312} 4 \mathrm{f}_{712}$ & $3^{\mathrm{e}}$ & $1.72574 \mathrm{E}+03$ & $1.72798 \mathrm{E}+03$ & --- & --- & --- & -- & --- \\
\hline 64 & $2 \mathrm{p}_{312} 4 \mathrm{f}_{512}$ & $2^{\mathrm{e}}$ & $1.72585 \mathrm{E}+03$ & $1.72807 \mathrm{E}+03$ & --- & --- & --- & --- & --- \\
\hline 65 & $2 \mathrm{p}_{312} 4 \mathrm{f}_{512}$ & $3^{e}$ & $1.72591 \mathrm{E}+03$ & $1.72810 \mathrm{E}+03$ & --- & --- & --- & --- & --- \\
\hline 66 & $2 \mathrm{p}_{312} 4 \mathrm{f}_{72}$ & $4^{\mathrm{e}}$ & $1.72613 \mathrm{E}+03$ & $1.72817 \mathrm{E}+03$ & --- & --- & --- & --- & --- \\
\hline 67 & $2 p_{12} 4 p_{12}$ & $0^{\mathrm{e}}$ & $1.72618 \mathrm{E}+03$ & $1.72838 \mathrm{E}+03$ & --- & --- & --- & --- & -- \\
\hline 68 & $2 \mathrm{p}_{12} 4 \mathrm{~d}_{312}$ & $2^{\circ}$ & $1.74573 \mathrm{E}+03$ & $1.74855 \mathrm{E}+03$ & --- & --- & --- & --- & -- \\
\hline 69 & $2 \mathrm{p}_{12} 4 \mathrm{~d}_{512}$ & $2^{\circ}$ & $1.74642 \mathrm{E}+03$ & $1.74941 \mathrm{E}+03$ & --- & --- & --- & --- & --- \\
\hline 70 & $2 \mathrm{p}_{12} 4 \mathrm{~d}_{512}$ & $3^{\circ}$ & $1.74687 \mathrm{E}+03$ & $1.74986 \mathrm{E}+03$ & --- & --- & -- & -- & --- \\
\hline 71 & $2 \mathrm{p}_{12} 4 \mathrm{~d}_{312}$ & $1^{\circ}$ & $1.74908 \mathrm{E}+03$ & $1.75109 \mathrm{E}+03$ & $1.75090 \mathrm{E}+03$ & --- & --- & --- & -- \\
\hline 72 & $2 p_{122} 4 f_{512}$ & $3^{\mathrm{e}}$ & $1.75784 \mathrm{E}+03$ & $1.76052 \mathrm{E}+03$ & --- & --- & --- & --- & --- \\
\hline 73 & $2 \mathrm{p}_{112} 4 \mathrm{f}_{712}$ & $4^{\mathrm{e}}$ & $1.75816 \mathrm{E}+03$ & $1.76073 \mathrm{E}+03$ & --- & --- & --- & --- & -- \\
\hline 74 & $2 \mathrm{p}_{112} 4 \mathrm{f}_{512}$ & $2^{\mathrm{e}}$ & $1.75818 \mathrm{E}+03$ & $1.76084 \mathrm{E}+03$ & --- & --- & --- & --- & --- \\
\hline 75 & $2 \mathrm{p}_{112} 4 \mathrm{f}_{712}$ & $3^{\mathrm{e}}$ & $1.75824 \mathrm{E}+03$ & $1.76085 \mathrm{E}+03$ & --- & --- & --- & -- & -- \\
\hline 76 & $2 \mathrm{p}_{312} 5 \mathrm{~s}_{112}$ & $2^{\circ}$ & $1.86014 \mathrm{E}+03$ & --- & --- & --- & --- & --- & --- \\
\hline 77 & $2 \mathrm{p}_{312} 5 \mathrm{~s}_{112}$ & $1^{\circ}$ & $1.86061 \mathrm{E}+03$ & --- & --- & --- & --- & --- & --- \\
\hline 78 & $2 \mathrm{~s}_{12} 4 \mathrm{~s}_{12}$ & $1^{\mathrm{e}}$ & $1.86083 \mathrm{E}+03$ & --- & --- & --- & -- & --- & --- \\
\hline 79 & $2 \mathrm{~s}_{12} 4 \mathrm{~s}_{112}$ & $0^{\mathrm{e}}$ & $1.86447 \mathrm{E}+03$ & --- & --- & --- & -- & --- & --- \\
\hline 80 & $2 \mathrm{p}_{312} 5 \mathrm{p}_{12}$ & $2^{\mathrm{e}}$ & $1.86954 \mathrm{E}+03$ & --- & --- & --- & --- & --- & --- \\
\hline 81 & $2 \mathrm{p}_{312} 5 \mathrm{p}_{12}$ & $1^{\mathrm{e}}$ & $1.86965 \mathrm{E}+03$ & --- & --- & --- & -- & -- & --- \\
\hline 82 & $2 p_{312} 5 p_{312}$ & $3^{\mathrm{e}}$ & $1.87079 \mathrm{E}+03$ & --- & --- & --- & --- & --- & -- \\
\hline 83 & $2 \mathrm{p}_{312} 5 \mathrm{p}_{312}$ & $1^{\mathrm{e}}$ & $1.87117 \mathrm{E}+03$ & --- & --- & --- & --- & --- & --- \\
\hline 84 & $2 \mathrm{p}_{312} 5 \mathrm{p}_{312}$ & $2^{\mathrm{e}}$ & $1.87148 \mathrm{E}+03$ & --- & --- & --- & --- & --- & -- \\
\hline 85 & $2 \mathrm{p}_{312} 5 \mathrm{p}_{312}$ & $0^{\mathrm{e}}$ & $1.87450 \mathrm{E}+03$ & --- & --- & --- & --- & -- & --- \\
\hline 86 & $2 \mathrm{~s}_{112} 4 \mathrm{p}_{112}$ & $0^{\circ}$ & $1.87921 \mathrm{E}+03$ & --- & --- & --- & --- & --- & --- \\
\hline 87 & $2 \mathrm{~s}_{122} 4 \mathrm{p}_{112}$ & $1^{\circ}$ & $1.87983 \mathrm{E}+03$ & --- & --- & --- & --- & --- & --- \\
\hline 88 & $2 \mathrm{p}_{312} 5 \mathrm{~d}_{312}$ & $0^{\circ}$ & $1.88142 \mathrm{E}+03$ & --- & --- & --- & --- & --- & --- \\
\hline 89 & $2 \mathrm{p}_{312} 5 \mathrm{~d}_{312}$ & $1^{\circ}$ & $1.88158 \mathrm{E}+03$ & --- & $1.88679 \mathrm{E}+03$ & --- & --- & --- & -- \\
\hline 90 & $2 \mathrm{p}_{312} 5 \mathrm{~d}_{512}$ & $2^{\circ}$ & $1.88163 \mathrm{E}+03$ & --- & --- & --- & --- & --- & --- \\
\hline 91 & $2 \mathrm{p}_{312} 5 \mathrm{~d}_{312}$ & $3^{\circ}$ & $1.88172 \mathrm{E}+03$ & --- & --- & --- & --- & --- & -- \\
\hline 92 & $2 \mathrm{p}_{312} 5 \mathrm{~d}_{512}$ & $4^{\circ}$ & $1.88173 \mathrm{E}+03$ & --- & --- & --- & --- & --- & -- \\
\hline 93 & $2 \mathrm{p}_{312} 5 \mathrm{~d}_{312}$ & $2^{\circ}$ & $1.88217 \mathrm{E}+03$ & --- & -- & -- & -- & -- & -- \\
\hline 94 & $2 \mathrm{p}_{312} 5 \mathrm{~d}_{512}$ & $3^{\circ}$ & $1.88255 \mathrm{E}+03$ & --- & --- & --- & --- & --- & --- \\
\hline 95 & $2 \mathrm{~s}_{12} 4 \mathrm{p}_{312}$ & $2^{\circ}$ & $1.88335 \mathrm{E}+03$ & --- & --- & -- & -- & -- & -- \\
\hline 96 & $2 \mathrm{~s}_{122} 4 \mathrm{p}_{312}$ & $1^{\circ}$ & $1.88338 \mathrm{E}+03$ & --- & $1.88679 \mathrm{E}+03$ & --- & --- & --- & --- \\
\hline 97 & $2 \mathrm{p}_{312} 5 \mathrm{~d}_{512}$ & $1^{\circ}$ & $1.88476 \mathrm{E}+03$ & --- & --- & --- & --- & --- & --- \\
\hline 98 & $2 \mathrm{p}_{312} 5 \mathrm{f}_{512}$ & $1^{\mathrm{e}}$ & $1.88719 \mathrm{E}+03$ & --- & --- & -- & -- & --- & -- \\
\hline 99 & $2 \mathrm{p}_{312} 5 \mathrm{f}_{512}$ & $2^{\mathrm{e}}$ & $1.88742 \mathrm{E}+03$ & --- & --- & --- & --- & --- & --- \\
\hline 100 & $2 \mathrm{p}_{312} 5 \mathrm{f}_{512}$ & $4^{\mathrm{e}}$ & $1.88754 \mathrm{E}+03$ & --- & --- & -- & -- & -- & -- \\
\hline 101 & $2 \mathrm{p}_{312} 5 \mathrm{f}_{712}$ & $5^{\mathrm{e}}$ & $1.88760 \mathrm{E}+03$ & --- & --- & -- & -- & --- & --- \\
\hline
\end{tabular}




\section{Continued}

\begin{tabular}{|c|c|c|c|c|c|c|c|c|c|}
\hline 102 & $2 \mathrm{p}_{312} 5 \mathrm{f}_{712}$ & $3^{\mathrm{e}}$ & $1.88780 \mathrm{E}+03$ & --- & --- & --- & -- & --- & -- \\
\hline 103 & $2 \mathrm{p}_{312} 5 \mathrm{~g}_{72}$ & $2^{\circ}$ & $1.88786 \mathrm{E}+03$ & --- & --- & --- & --- & --- & --- \\
\hline 104 & $2 \mathrm{p}_{312} 5 \mathrm{~g}_{912}$ & $3^{\circ}$ & $1.88795 \mathrm{E}+03$ & --- & --- & --- & --- & --- & -- \\
\hline 105 & $2 p_{312} 5 f_{512}$ & $3^{\mathrm{e}}$ & $1.88795 \mathrm{E}+03$ & --- & --- & --- & -- & --- & --- \\
\hline 106 & $2 \mathrm{p}_{312} 5 \mathrm{f}_{72}$ & $2^{\mathrm{e}}$ & $1.88797 \mathrm{E}+03$ & --- & --- & --- & --- & --- & -- \\
\hline 107 & $2 \mathrm{p}_{312} 5 \mathrm{f}_{712}$ & $4^{e}$ & $1.88808 \mathrm{E}+03$ & -- & --- & -- & -- & --- & -- \\
\hline 108 & $2 \mathrm{p}_{312} 5 \mathrm{~g}_{72}$ & $5^{\circ}$ & $1.88817 \mathrm{E}+03$ & --- & --- & --- & -- & --- & --- \\
\hline 109 & $2 \mathrm{p}_{312} 5 \mathrm{~g}_{712}$ & $3^{\circ}$ & $1.88824 \mathrm{E}+03$ & -- & --- & --- & -- & --- & --- \\
\hline 110 & $2 \mathrm{p}_{312} 5 \mathrm{~g}_{912}$ & $6^{\circ}$ & $1.88825 \mathrm{E}+03$ & --- & --- & -- & -- & --- & -- \\
\hline 111 & $2 \mathrm{p}_{312} 5 \mathrm{~g}_{912}$ & $4^{\circ}$ & $1.88830 \mathrm{E}+03$ & -- & --- & -- & --- & --- & -- \\
\hline 112 & $2 \mathrm{p}_{312} 5 \mathrm{~g}_{72}$ & $4^{\circ}$ & $1.88842 \mathrm{E}+03$ & -- & --- & -- & --- & --- & -- \\
\hline 113 & $2 \mathrm{p}_{312} 5 \mathrm{~g}_{912}$ & $5^{\circ}$ & $1.88849 \mathrm{E}+03$ & --- & --- & --- & --- & --- & --- \\
\hline 114 & $2 \mathrm{p}_{12} 5 \mathrm{~s}_{112}$ & $0^{\circ}$ & $1.89308 \mathrm{E}+03$ & --- & --- & -- & -- & --- & -- \\
\hline 115 & $2 \mathrm{p}_{12} 5 \mathrm{~s}_{112}$ & $1^{\circ}$ & $1.89329 \mathrm{E}+03$ & --- & --- & -- & --- & --- & -- \\
\hline 116 & $2 \mathrm{p}_{112} 5 \mathrm{p}_{12}$ & $1^{\mathrm{e}}$ & $1.90155 \mathrm{E}+03$ & -- & --- & -- & -- & --- & -- \\
\hline 117 & $2 \mathrm{p}_{12} 5 \mathrm{p}_{312}$ & $2^{\mathrm{e}}$ & $1.90316 \mathrm{E}+03$ & -- & --- & -- & -- & --- & -- \\
\hline 118 & $2 \mathrm{p}_{112} 5 \mathrm{p}_{312}$ & $1^{\mathrm{e}}$ & $1.90352 \mathrm{E}+03$ & --- & --- & -- & --- & --- & -- \\
\hline 119 & $2 \mathrm{p}_{112} 5 \mathrm{p}_{112}$ & $0^{\mathrm{e}}$ & $1.90422 \mathrm{E}+03$ & -- & --- & -- & -- & --- & -- \\
\hline 120 & $2 \mathrm{~s}_{112} 4 \mathrm{~d}_{512}$ & $3^{\mathrm{e}}$ & $1.90492 \mathrm{E}+03$ & -- & --- & -- & -- & --- & -- \\
\hline 121 & $2 \mathrm{~s}_{112} 4 \mathrm{~d}_{312}$ & $1^{\mathrm{e}}$ & $1.90493 \mathrm{E}+03$ & --- & --- & -- & -- & --- & --- \\
\hline 122 & $2 \mathrm{~s}_{12} 4 \mathrm{~d}_{312}$ & $2^{\mathrm{e}}$ & $1.90495 \mathrm{E}+03$ & -- & --- & -- & -- & --- & -- \\
\hline 123 & $2 \mathrm{~s}_{12} 4 \mathrm{~d}_{312}$ & $2^{\mathrm{e}}$ & $1.90740 \mathrm{E}+03$ & --- & --- & -- & --- & --- & -- \\
\hline 124 & $2 \mathrm{p}_{12} 5 \mathrm{~d}_{312}$ & $2^{\circ}$ & $1.91418 \mathrm{E}+03$ & --- & --- & -- & --- & --- & -- \\
\hline 125 & $2 \mathrm{p}_{112} 5 \mathrm{~d}_{512}$ & $2^{\circ}$ & $1.91454 \mathrm{E}+03$ & -- & --- & -- & --- & --- & -- \\
\hline 126 & $2 \mathrm{p}_{112} 5 \mathrm{~d}_{512}$ & $3^{\circ}$ & $1.91471 \mathrm{E}+03$ & -- & --- & -- & --- & --- & -- \\
\hline 127 & $2 \mathrm{~s}_{12} 4 \mathrm{f}_{512}$ & $3^{\circ}$ & $1.91553 \mathrm{E}+03$ & -- & --- & -- & --- & --- & -- \\
\hline 128 & $2 \mathrm{~s}_{12} 4 \mathrm{f}_{712}$ & $4^{\circ}$ & $1.91561 \mathrm{E}+03$ & -- & --- & -- & -- & --- & -- \\
\hline 129 & $2 \mathrm{p}_{12} 5 \mathrm{~d}_{312}$ & $1^{\circ}$ & $1.91567 \mathrm{E}+03$ & --- & $1.91779 \mathrm{E}+03$ & -- & --- & --- & -- \\
\hline 130 & $2 \mathrm{~s}_{12} 4 \mathrm{f}_{512}$ & $2^{\circ}$ & $1.91624 \mathrm{E}+03$ & -- & --- & -- & --- & --- & -- \\
\hline 131 & $2 \mathrm{~s}_{12} 4 \mathrm{f}_{712}$ & $3^{\circ}$ & $1.91667 \mathrm{E}+03$ & -- & --- & -- & -- & -- & -- \\
\hline 132 & $2 p_{12} 5 f_{512}$ & $3^{\mathrm{e}}$ & $1.92023 \mathrm{E}+03$ & --- & --- & --- & --- & -- & -- \\
\hline 133 & $2 \mathrm{p}_{12} 5 \mathrm{f}_{72}$ & $4^{\mathrm{e}}$ & $1.92040 \mathrm{E}+03$ & -- & --- & -- & -- & -- & -- \\
\hline 134 & $2 \mathrm{p}_{122} 5 \mathrm{f}_{512}$ & $2^{\mathrm{e}}$ & $1.92046 \mathrm{E}+03$ & -- & --- & -- & -- & -- & -- \\
\hline 135 & $2 \mathrm{p}_{122} 5 \mathrm{f}_{72}$ & $3^{\mathrm{e}}$ & $1.92049 \mathrm{E}+03$ & -- & --- & -- & --- & -- & -- \\
\hline 136 & $2 \mathrm{p}_{112} 5 \mathrm{~g}_{712}$ & $4^{\circ}$ & $1.92087 \mathrm{E}+03$ & -- & --- & -- & --- & --- & -- \\
\hline 137 & $2 \mathrm{p}_{12} 5 \mathrm{~g}_{912}$ & $5^{\circ}$ & $1.92095 \mathrm{E}+03$ & -- & --- & -- & --- & -- & -- \\
\hline 138 & $2 \mathrm{p}_{112} 5 \mathrm{~g}_{72}$ & $3^{\circ}$ & $1.92159 \mathrm{E}+03$ & --- & --- & --- & --- & --- & -- \\
\hline 139 & $2 \mathrm{p}_{112} 5 \mathrm{~g}_{912}$ & $4^{\circ}$ & $1.92168 \mathrm{E}+03$ & -- & --- & -- & --- & --- & -- \\
\hline 140 & $2 \mathrm{p}_{312} 6 \mathrm{~s}_{112}$ & $2^{\circ}$ & $1.96032 \mathrm{E}+03$ & --- & --- & --- & --- & -- & -- \\
\hline 141 & $2 \mathrm{p}_{312} 6 \mathrm{~s}_{112}$ & $1^{\circ}$ & $1.96055 \mathrm{E}+03$ & --- & --- & --- & -- & --- & --- \\
\hline
\end{tabular}




\section{Continued}

\begin{tabular}{|c|c|c|c|c|c|c|c|c|c|}
\hline 142 & $2 \mathrm{p}_{312} 6 \mathrm{p}_{112}$ & $1^{\mathrm{e}}$ & $1.96543 \mathrm{E}+03$ & --- & --- & --- & -- & --- & -- \\
\hline 143 & $2 \mathrm{p}_{312} 6 \mathrm{p}_{112}$ & $2^{\mathrm{e}}$ & $1.96562 \mathrm{E}+03$ & --- & --- & --- & -- & --- & --- \\
\hline 144 & $2 \mathrm{p}_{312} 6 \mathrm{p}_{312}$ & $3^{\mathrm{e}}$ & $1.96636 \mathrm{E}+03$ & -- & --- & -- & -- & --- & --- \\
\hline 145 & $2 \mathrm{p}_{312} 6 \mathrm{p}_{312}$ & $1^{\mathrm{e}}$ & $1.96644 \mathrm{E}+03$ & --- & --- & -- & -- & --- & --- \\
\hline 146 & $2 \mathrm{p}_{312} 6 \mathrm{p}_{312}$ & $2^{\mathrm{e}}$ & $1.96670 \mathrm{E}+03$ & --- & --- & --- & -- & --- & --- \\
\hline 147 & $2 \mathrm{p}_{312} 6 \mathrm{p}_{312}$ & $0^{\mathrm{e}}$ & $1.96836 \mathrm{E}+03$ & --- & --- & --- & --- & --- & -- \\
\hline 148 & $2 \mathrm{p}_{312} 6 \mathrm{~d}_{312}$ & $0^{\circ}$ & $1.97209 \mathrm{E}+03$ & --- & --- & --- & -- & --- & --- \\
\hline 149 & $2 \mathrm{p}_{312} 6 \mathrm{~d}_{312}$ & $1^{\circ}$ & $1.97229 \mathrm{E}+03$ & --- & --- & --- & -- & --- & -- \\
\hline 150 & $2 \mathrm{p}_{312} 6 \mathrm{~d}_{312}$ & $3^{\circ}$ & $1.97253 \mathrm{E}+03$ & --- & --- & --- & --- & --- & --- \\
\hline 151 & $2 \mathrm{p}_{312} 6 \mathrm{~d}_{512}$ & $4^{\circ}$ & $1.97257 \mathrm{E}+03$ & --- & --- & --- & --- & --- & -- \\
\hline 152 & $2 \mathrm{p}_{312} 6 \mathrm{~d}_{312}$ & $2^{\circ}$ & $1.97259 \mathrm{E}+03$ & --- & --- & --- & --- & --- & -- \\
\hline 153 & $2 \mathrm{p}_{312} 6 \mathrm{~d}_{512}$ & $2^{\circ}$ & $1.97279 \mathrm{E}+03$ & --- & --- & --- & --- & --- & --- \\
\hline 154 & $2 \mathrm{p}_{312} 6 \mathrm{~d}_{512}$ & $3^{\circ}$ & $1.97299 \mathrm{E}+03$ & -- & --- & -- & -- & --- & -- \\
\hline 155 & $2 \mathrm{p}_{312} 6 \mathrm{~d}_{512}$ & $1^{\circ}$ & $1.97404 \mathrm{E}+03$ & --- & $1.97680 \mathrm{E}+03$ & --- & --- & --- & --- \\
\hline 156 & $2 \mathrm{p}_{312} 6 \mathrm{f}_{512}$ & $1^{\mathrm{e}}$ & $1.97568 \mathrm{E}+03$ & -- & --- & -- & -- & --- & -- \\
\hline 157 & $2 \mathrm{p}_{312} 6 \mathrm{f}_{512}$ & $4^{e}$ & $1.97579 \mathrm{E}+03$ & --- & --- & --- & -- & --- & -- \\
\hline 158 & $2 \mathrm{p}_{312} 6 \mathrm{f}_{512}$ & $2^{\mathrm{e}}$ & $1.97580 \mathrm{E}+03$ & --- & --- & --- & --- & --- & --- \\
\hline 159 & $2 \mathrm{p}_{312} 6 \mathrm{f}_{72}$ & $5^{\mathrm{e}}$ & $1.97583 \mathrm{E}+03$ & -- & --- & -- & -- & --- & -- \\
\hline 160 & $2 \mathrm{p}_{312} 6 \mathrm{f}_{512}$ & $3^{\mathrm{e}}$ & $1.97597 \mathrm{E}+03$ & --- & --- & --- & -- & --- & -- \\
\hline 161 & $2 \mathrm{p}_{312} 6 \mathrm{f}_{712}$ & $3^{\mathrm{e}}$ & $1.97602 \mathrm{E}+03$ & --- & --- & --- & -- & --- & -- \\
\hline 162 & $2 \mathrm{p}_{312} 6 \mathrm{p}_{72}$ & $2^{\mathrm{e}}$ & $1.97605 \mathrm{E}+03$ & --- & --- & --- & -- & --- & -- \\
\hline 163 & $2 p_{312} 6 f_{712}$ & $4^{e}$ & $1.97609 \mathrm{E}+03$ & --- & --- & --- & -- & --- & --- \\
\hline 164 & $2 p_{312} 6 g_{72}$ & $2^{\circ}$ & $1.97626 \mathrm{E}+03$ & --- & --- & --- & --- & --- & --- \\
\hline 165 & $2 \mathrm{p}_{312} 6 \mathrm{~g}_{712}$ & $5^{\circ}$ & $1.97626 \mathrm{E}+03$ & --- & --- & --- & --- & --- & -- \\
\hline 166 & $2 \mathrm{p}_{312} 6 \mathrm{~h}_{912}$ & $3^{\mathrm{e}}$ & $1.97627 \mathrm{E}+03$ & -- & --- & -- & -- & --- & -- \\
\hline 167 & $2 \mathrm{p}_{312} 6 \mathrm{~h}_{112}$ & $4^{\mathrm{e}}$ & $1.97630 \mathrm{E}+03$ & --- & --- & --- & -- & --- & -- \\
\hline 168 & $2 \mathrm{p}_{312} 6 \mathrm{~h}_{912}$ & $6^{\mathrm{e}}$ & $1.97630 \mathrm{E}+03$ & --- & --- & --- & -- & --- & -- \\
\hline 169 & $2 \mathrm{p}_{312} 6 \mathrm{~g}_{912}$ & $6^{\circ}$ & $1.97631 \mathrm{E}+03$ & --- & --- & --- & --- & --- & --- \\
\hline 170 & $2 \mathrm{p}_{312} 6 \mathrm{~g}_{912}$ & $3^{\circ}$ & $1.97631 \mathrm{E}+03$ & --- & --- & --- & --- & --- & -- \\
\hline 171 & $2 p_{312} 6 h_{1112}$ & $7^{e}$ & $1.97633 \mathrm{E}+03$ & --- & --- & --- & -- & -- & -- \\
\hline 172 & $2 \mathrm{p}_{312} 6 \mathrm{~h}_{912}$ & $4^{\circ}$ & $1.97635 \mathrm{E}+03$ & --- & --- & --- & --- & --- & --- \\
\hline 173 & $2 \mathrm{p}_{312} 6 \mathrm{~h}_{912}$ & $5^{\mathrm{e}}$ & $1.97637 \mathrm{E}+03$ & --- & --- & --- & -- & --- & -- \\
\hline 174 & $2 \mathrm{p}_{312} 6 \mathrm{~g}_{712}$ & $3^{\circ}$ & $1.97638 \mathrm{E}+03$ & --- & --- & --- & --- & --- & --- \\
\hline 175 & $2 \mathrm{p}_{312} 6 \mathrm{~h}_{1112}$ & $5^{\mathrm{e}}$ & $1.97638 \mathrm{E}+03$ & -- & --- & -- & -- & --- & -- \\
\hline 176 & $2 \mathrm{p}_{312} 6 \mathrm{~g}_{712}$ & $4^{\circ}$ & $1.97639 \mathrm{E}+03$ & --- & --- & --- & --- & --- & -- \\
\hline 177 & $2 \mathrm{p}_{312} 6 \mathrm{~g}_{712}$ & $6^{\mathrm{e}}$ & $1.97641 \mathrm{E}+03$ & --- & --- & --- & -- & -- & -- \\
\hline 178 & $2 \mathrm{p}_{312} 6 \mathrm{~g}_{912}$ & $4^{\circ}$ & $1.97642 \mathrm{E}+03$ & --- & --- & --- & --- & --- & --- \\
\hline 179 & $2 \mathrm{p}_{312} 6 \mathrm{~g}_{912}$ & $5^{\circ}$ & $1.97644 \mathrm{E}+03$ & --- & --- & --- & --- & --- & --- \\
\hline 180 & $2 \mathrm{p}_{12} 6 \mathrm{~s}_{112}$ & $0^{\circ}$ & $1.99290 \mathrm{E}+03$ & --- & --- & --- & --- & --- & --- \\
\hline 181 & $2 \mathrm{p}_{12} 6 \mathrm{~s}_{112}$ & $1^{\circ}$ & $1.99302 \mathrm{E}+03$ & -- & --- & -- & -- & -- & -- \\
\hline
\end{tabular}




\section{Continued}

\begin{tabular}{|c|c|c|c|c|c|c|c|c|c|}
\hline 182 & $2 \mathrm{p}_{12} 6 \mathrm{p}_{12}$ & $1^{\mathrm{e}}$ & $1.99809 \mathrm{E}+03$ & --- & --- & --- & -- & --- & -- \\
\hline 183 & $2 p_{12} 6 p_{312}$ & $1^{\mathrm{e}}$ & $1.99900 \mathrm{E}+03$ & --- & --- & --- & --- & --- & --- \\
\hline 184 & $2 p_{12} 6 p_{312}$ & $2^{\mathrm{e}}$ & $1.99910 \mathrm{E}+03$ & --- & --- & --- & --- & --- & -- \\
\hline 185 & $2 \mathrm{p}_{12} 6 \mathrm{p}_{12}$ & $0^{\mathrm{e}}$ & $1.99929 \mathrm{E}+03$ & --- & --- & --- & --- & --- & -- \\
\hline 186 & $2 \mathrm{p}_{122} 6 \mathrm{~d}_{312}$ & $2^{\circ}$ & $2.00505 \mathrm{E}+03$ & --- & --- & --- & --- & --- & -- \\
\hline 187 & $2 \mathrm{p}_{122} 6 \mathrm{~d}_{512}$ & $2^{\circ}$ & $2.00526 \mathrm{E}+03$ & -- & --- & -- & -- & --- & -- \\
\hline 188 & $2 \mathrm{p}_{122} 6 \mathrm{~d}_{512}$ & $3^{\circ}$ & $2.00538 \mathrm{E}+03$ & --- & --- & --- & --- & --- & -- \\
\hline 189 & $2 \mathrm{p}_{122} 6 \mathrm{~d}_{312}$ & $1^{\circ}$ & $2.00585 \mathrm{E}+03$ & --- & $2.00879 \mathrm{E}+03$ & --- & -- & --- & --- \\
\hline 190 & $2 p_{122} 6 f_{512}$ & $3^{\mathrm{e}}$ & $2.00844 \mathrm{E}+03$ & --- & --- & -- & -- & --- & -- \\
\hline 191 & $2 \mathrm{p}_{122} 6 \mathrm{f}_{72}$ & $3^{\mathrm{e}}$ & $2.00854 \mathrm{E}+03$ & -- & --- & -- & --- & --- & -- \\
\hline 192 & $2 \mathrm{p}_{122} 6 \mathrm{f}_{712}$ & $4^{\mathrm{e}}$ & $2.00854 \mathrm{E}+03$ & -- & --- & -- & -- & --- & -- \\
\hline 193 & $2 p_{12} 6 f_{512}$ & $2^{\mathrm{e}}$ & $2.00855 \mathrm{E}+03$ & --- & --- & --- & --- & --- & --- \\
\hline 194 & $2 \mathrm{p}_{122} 6 \mathrm{~g}_{72}$ & $4^{\circ}$ & $2.00891 \mathrm{E}+03$ & --- & --- & -- & -- & --- & -- \\
\hline 195 & $2 \mathrm{p}_{12} 6 \mathrm{~h}_{912}$ & $4^{\mathrm{e}}$ & $2.00892 \mathrm{E}+03$ & --- & --- & -- & -- & --- & -- \\
\hline 196 & $2 p_{12} 6 \mathrm{~s}_{\mathrm{g} 72}$ & $3^{\circ}$ & $2.00893 \mathrm{E}+03$ & -- & --- & -- & --- & --- & -- \\
\hline 197 & $2 \mathrm{p}_{12} 6 \mathrm{~h}_{912}$ & $5^{\mathrm{e}}$ & $2.00894 \mathrm{E}+03$ & -- & --- & -- & -- & --- & -- \\
\hline 198 & $2 \mathrm{p}_{112} 6 \mathrm{~h}_{1112}$ & $5^{\mathrm{e}}$ & $2.00896 \mathrm{E}+03$ & --- & --- & -- & --- & --- & -- \\
\hline 199 & $2 \mathrm{p}_{122} 6 \mathrm{~g}_{912}$ & $5^{\circ}$ & $2.00896 \mathrm{E}+03$ & --- & --- & -- & -- & --- & -- \\
\hline 200 & $2 \mathrm{p}_{112} 6 \mathrm{~h}_{112}$ & $6^{\mathrm{e}}$ & $2.00897 \mathrm{E}+03$ & -- & --- & -- & -- & --- & -- \\
\hline 201 & $2 \mathrm{p}_{122} 6 \mathrm{~g}_{912}$ & $4^{\circ}$ & $2.00897 \mathrm{E}+03$ & --- & --- & -- & --- & --- & --- \\
\hline 202 & $2 \mathrm{~s}_{12} 5 \mathrm{~s}_{12}$ & $1^{\mathrm{e}}$ & $2.05136 \mathrm{E}+03$ & -- & --- & -- & -- & --- & -- \\
\hline 203 & $2 \mathrm{~s}_{12} 5 \mathrm{~s}_{112}$ & $0^{\mathrm{e}}$ & $2.05274 \mathrm{E}+03$ & --- & --- & -- & --- & --- & -- \\
\hline 204 & $2 \mathrm{~s}_{12} 5 \mathrm{p}_{12}$ & $0^{\circ}$ & $2.06056 \mathrm{E}+03$ & --- & --- & -- & --- & --- & -- \\
\hline 205 & $2 \mathrm{~s}_{12} 5 \mathrm{p}_{12}$ & $1^{\circ}$ & $2.06068 \mathrm{E}+03$ & --- & --- & --- & --- & --- & --- \\
\hline 206 & $2 \mathrm{~s}_{12} 5 \mathrm{p}_{312}$ & $2^{\circ}$ & $2.06209 \mathrm{E}+03$ & --- & --- & --- & --- & --- & --- \\
\hline 207 & $2 \mathrm{~s}_{12} 5 \mathrm{p}_{312}$ & $1^{\circ}$ & $2.06247 \mathrm{E}+03$ & --- & --- & --- & --- & --- & -- \\
\hline 208 & $2 \mathrm{~s}_{12} 5 \mathrm{~d}_{312}$ & $1^{\mathrm{e}}$ & $2.07254 \mathrm{E}+03$ & -- & --- & -- & -- & --- & -- \\
\hline 209 & $2 \mathrm{~s}_{12} 5 \mathrm{~d}_{312}$ & $2^{\mathrm{e}}$ & $2.07264 \mathrm{E}+03$ & --- & --- & -- & --- & --- & -- \\
\hline 210 & $2 \mathrm{~s}_{122} 5 \mathrm{~d}_{512}$ & $3^{\mathrm{e}}$ & $2.07286 \mathrm{E}+03$ & -- & --- & -- & --- & --- & -- \\
\hline 211 & $2 \mathrm{~s}_{12} 5 \mathrm{~d}_{512}$ & $2^{\mathrm{e}}$ & $2.07389 \mathrm{E}+03$ & -- & --- & -- & -- & -- & -- \\
\hline 212 & $2 \mathrm{~s}_{12} 5 \mathrm{f}_{512}$ & $2^{\circ}$ & $2.07841 \mathrm{E}+03$ & --- & --- & --- & --- & -- & -- \\
\hline 213 & $2 \mathrm{~s}_{12} 5 \mathrm{f}_{512}$ & $3^{\circ}$ & $2.07844 \mathrm{E}+03$ & -- & --- & -- & -- & -- & -- \\
\hline 214 & $2 \mathrm{~s}_{12} 5 \mathrm{f}_{712}$ & $4^{\circ}$ & $2.07853 \mathrm{E}+03$ & -- & --- & -- & --- & -- & -- \\
\hline 215 & $2 \mathrm{~s}_{12} 5 \mathrm{f}_{712}$ & $3^{\circ}$ & $2.07871 \mathrm{E}+03$ & -- & --- & -- & -- & -- & -- \\
\hline 216 & $2 \mathrm{~s}_{122} 5 \mathrm{~g}_{72}$ & $4^{\mathrm{e}}$ & $2.07901 \mathrm{E}+03$ & -- & --- & --- & --- & -- & --- \\
\hline 217 & $2 \mathrm{~s}_{12} 5 \mathrm{~g}_{72}$ & $3^{\mathrm{e}}$ & $2.07901 \mathrm{E}+03$ & --- & --- & --- & --- & -- & --- \\
\hline 218 & $2 \mathrm{~s}_{12} 5 \mathrm{~g}_{912}$ & $5^{\mathrm{e}}$ & $2.07909 \mathrm{E}+03$ & -- & --- & -- & -- & --- & -- \\
\hline 219 & $2 \mathrm{~s}_{12} 5 \mathrm{~g}_{912}$ & $4^{e}$ & $2.07909 \mathrm{E}+03$ & -- & --- & -- & -- & --- & -- \\
\hline 220 & $2 \mathrm{~s}_{12} 6 \mathrm{~s}_{12}$ & $1^{\mathrm{e}}$ & $2.15120 \mathrm{E}+03$ & --- & --- & --- & -- & -- & -- \\
\hline 221 & $2 \mathrm{~s}_{12} 6 \mathrm{~s}_{12}$ & $0^{\mathrm{e}}$ & $2.15196 \mathrm{E}+03$ & --- & --- & -- & -- & --- & -- \\
\hline
\end{tabular}




\section{Continued}

\begin{tabular}{|c|c|c|c|c|c|c|c|c|c|}
\hline 222 & $2 \mathrm{~s}_{112} 6 \mathrm{p}_{122}$ & $0^{\circ}$ & $2.15644 \mathrm{E}+03$ & --- & --- & --- & --- & --- & -- \\
\hline 223 & $2 s_{112} 6 p_{112}$ & $1^{\circ}$ & $2.15652 \mathrm{E}+03$ & --- & --- & --- & --- & --- & -- \\
\hline 224 & $2 \mathrm{~s}_{112} 6 \mathrm{p}_{312}$ & $2^{\circ}$ & $2.15735 \mathrm{E}+03$ & --- & --- & --- & --- & --- & -- \\
\hline 225 & $2 \mathrm{~s}_{112} 6 \mathrm{p}_{312}$ & $1^{\circ}$ & $2.15755 \mathrm{E}+03$ & --- & --- & --- & --- & --- & --- \\
\hline 226 & $2 \mathrm{~s}_{112} 6 \mathrm{~d}_{312}$ & $1^{\mathrm{e}}$ & $2.16329 \mathrm{E}+03$ & --- & --- & --- & --- & --- & --- \\
\hline 227 & $2 \mathrm{~s}_{112} 6 \mathrm{~d}_{312}$ & $2^{\mathrm{e}}$ & $2.16336 \mathrm{E}+03$ & --- & --- & --- & --- & --- & --- \\
\hline 228 & $2 \mathrm{~s}_{112} 6 \mathrm{~d}_{512}$ & $3^{\mathrm{e}}$ & $2.16349 \mathrm{E}+03$ & --- & --- & --- & --- & --- & --- \\
\hline 229 & $2 \mathrm{~s}_{112} 6 \mathrm{~d}_{512}$ & $2^{\mathrm{e}}$ & $2.16403 E+03$ & --- & --- & -- & --- & --- & -- \\
\hline 230 & $2 \mathrm{~s}_{112} 6 \mathrm{f}_{512}$ & $2^{\circ}$ & $2.16658 \mathrm{E}+03$ & --- & -- & --- & --- & --- & -- \\
\hline 231 & $2 \mathrm{~s}_{112} 6 \mathrm{f}_{512}$ & $3^{\circ}$ & $2.16660 \mathrm{E}+03$ & --- & --- & --- & --- & --- & --- \\
\hline 232 & $2 \mathrm{~s}_{112} 6 \mathrm{f}_{712}$ & $4^{\circ}$ & $2.16665 \mathrm{E}+03$ & --- & -- & --- & --- & --- & -- \\
\hline 233 & $2 \mathrm{~s}_{112} 6 \mathrm{f}_{712}$ & $3^{\circ}$ & $2.16676 \mathrm{E}+03$ & --- & -- & --- & --- & --- & -- \\
\hline 234 & $2 \mathrm{~s}_{112} 6 \mathrm{~g}_{712}$ & $3^{\mathrm{e}}$ & $2.16703 \mathrm{E}+03$ & --- & --- & --- & --- & --- & -- \\
\hline 235 & $2 s_{112} 6 g_{712}$ & $4^{\mathrm{e}}$ & $2.16703 \mathrm{E}+03$ & --- & --- & -- & --- & --- & --- \\
\hline 236 & $2 \mathrm{~s}_{112} 6 \mathrm{~h}_{912}$ & $5^{\circ}$ & $2.16705 E+03$ & --- & --- & --- & --- & --- & --- \\
\hline 237 & $2 \mathrm{~s}_{112} 6 \mathrm{~h}_{912}$ & $4^{\circ}$ & $2.16705 \mathrm{E}+03$ & --- & --- & --- & --- & --- & --- \\
\hline 238 & $2 \mathrm{~s}_{112} 6 \mathrm{~g}_{912}$ & $5^{\mathrm{e}}$ & $2.16708 \mathrm{E}+03$ & --- & --- & --- & --- & --- & --- \\
\hline 239 & $2 \mathrm{~s}_{112} 6 \mathrm{~h}_{1112}$ & $6^{\circ}$ & $2.16708 \mathrm{E}+03$ & --- & --- & -- & --- & --- & -- \\
\hline 240 & $2 \mathrm{~s}_{12} 6 \mathrm{~h}_{1112}$ & $5^{\circ}$ & $2.16708 \mathrm{E}+03$ & --- & --- & --- & --- & --- & -- \\
\hline 241 & $2 \mathrm{~s}_{112} 6 \mathrm{~g}_{912}$ & $4^{\mathrm{e}}$ & $2.16708 \mathrm{E}+03$ & --- & --- & --- & --- & --- & -- \\
\hline
\end{tabular}

Index: Level index. State Configuration: The configuration to which the level belongs (jj-coupling). $\mathbf{J}^{\pi}: \mathbf{J}$ is the Total angular momentum, $\pi$ is the Parity. FAC: Energies from the FAC code (our calculation). COWAN: Energies from the COWAN code (also calculated). NIST: National Institute of Standards and Technology, http://www.nist.gov [24]. Exp.: Energies calculated experimentally [23]. CIV3: Energies from the CIV3 code [22]. MCDF: Energies from the MCDF code [23]. RCI: Energies from the RCI code [23].

Table 2. Comparison between some weighted oscillator strengths, and transition probabilities for E1 transitions of Ge XXIII.

\begin{tabular}{|c|c|c|c|c|c|c|c|c|}
\hline $\mathrm{j}$ & $\mathrm{i}$ & $\operatorname{gf}(\mathrm{FAC})$ & $\operatorname{gf}(\mathrm{COWAN})$ & gf (CIV3) & gf (RCI) & $\mathrm{A}_{\mathrm{ji}}\left(\mathrm{s}^{-1}\right)(\mathrm{FAC})$ & $\mathrm{A}_{\mathrm{ji}}\left(\mathrm{s}^{-1}\right)(\mathrm{COWAN})$ & $\mathrm{A}_{\mathrm{ji}}\left(\mathrm{s}^{-1}\right)(\mathrm{CIV} 3)$ \\
\hline 3 & 1 & $1.29 \mathrm{E}-01$ & $1.0139 \mathrm{E}-01$ & $1.280 \mathrm{E}-01$ & --- & $2.86 \mathrm{E}+12$ & $2.2503 \mathrm{E}+12$ & $2.84 \mathrm{E}+12$ \\
\hline 5 & 1 & $8.88 \mathrm{E}-02$ & $7.0307 \mathrm{E}-02$ & $8.480 \mathrm{E}-02$ & ---- & $2.07 \mathrm{E}+12$ & $1.6400 \mathrm{E}+12$ & $1.1868 \mathrm{E}+12$ \\
\hline 6 & 2 & $2.26 \mathrm{E}-01$ & $2.5061 \mathrm{E}-01$ & $2.257 \mathrm{E}-01$ & 0.2685 & $5.96 \mathrm{E}+09$ & $6.5800 \mathrm{E}+09$ & $5.9267 \mathrm{E}+09$ \\
\hline 7 & 2 & $2.13 \mathrm{E}-01$ & $2.1330 \mathrm{E}-01$ & $2.113 \mathrm{E}-01$ & 0.2246 & $3.96 \mathrm{E}+09$ & $3.9660 \mathrm{E}+09$ & $3.9080 \mathrm{E}+09$ \\
\hline 7 & 3 & $2.15 \mathrm{E}-01$ & $2.2961 \mathrm{E}-01$ & $2.185 \mathrm{E}-01$ & 0.2204 & $3.51 \mathrm{E}+09$ & $3.7720 \mathrm{E}+09$ & $3.5940 \mathrm{E}+09$ \\
\hline 8 & 2 & $7.00 \mathrm{E}-01$ & $7.2277 \mathrm{E}-01$ & $7.002 \mathrm{E}-01$ & 0.7275 & $1.18 \mathrm{E}+10$ & $1.2177 \mathrm{E}+10$ & $1.1760 \mathrm{E}+10$ \\
\hline 9 & 2 & $4.53 \mathrm{E}-04$ & $1.1885 \mathrm{E}-04$ & $4.000 \mathrm{E}-04$ & 0.0001 & $1.85 \mathrm{E}+07$ & $4.7833 \mathrm{E}+06$ & $1.5900 \mathrm{E}+07$ \\
\hline 9 & 3 & $2.84 \mathrm{E}-01$ & $2.9512 \mathrm{E}-01$ & $2.827 \mathrm{E}-01$ & 0.2914 & $1.03 \mathrm{E}+10$ & $1.0657 \mathrm{E}+10$ & $1.0260 \mathrm{E}+10$ \\
\hline 10 & 2 & $2.91 \mathrm{E}-01$ & $2.9107 \mathrm{E}-01$ & $2.923 \mathrm{E}-01$ & 0.3092 & $8.02 \mathrm{E}+09$ & $7.9700 \mathrm{E}+09$ & $7.9360 \mathrm{E}+09$ \\
\hline 10 & 3 & $2.52 \mathrm{E}-01$ & $2.4604 \mathrm{E}-01$ & $2.480 \mathrm{E}-01$ & 0.2431 & $6.27 \mathrm{E}+09$ & $6.0900 \mathrm{E}+09$ & $6.1160 \mathrm{E}+09$ \\
\hline 10 & 5 & $6.21 \mathrm{E}-04$ & $8.1658 \mathrm{E}-04$ & $5.000 \mathrm{E}-04$ & --- & $2.70 \mathrm{E}+06$ & $3.4520 \mathrm{E}+06$ & $2.3540 \mathrm{E}+06$ \\
\hline 11 & 3 & $1.08 \mathrm{E}-01$ & $1.0495 \mathrm{E}-01$ & $1.102 \mathrm{E}-01$ & 0.0930 & $1.98 \mathrm{E}+10$ & $1.9050 \mathrm{E}+10$ & $2.0180 \mathrm{E}+10$ \\
\hline 11 & 5 & $1.26 \mathrm{E}-02$ & $1.1588 \mathrm{E}-02$ & $1.130 \mathrm{E}-02$ & ---- & $6.31 \mathrm{E}+08$ & $5.5900 \mathrm{E}+08$ & $5.6060 \mathrm{E}+08$ \\
\hline 12 & 2 & $6.08 \mathrm{E}-04$ & $4.2267 \mathrm{E}-04$ & $7.000 \mathrm{E}-04$ & 0.0000 & $5.31 \mathrm{E}+07$ & $3.6867 \mathrm{E}+07$ & $6.0800 \mathrm{E}+07$ \\
\hline 12 & 3 & $5.51 \mathrm{E}-04$ & $2.8249 \mathrm{E}-04$ & $7.000 \mathrm{E}-04$ & 0.0076 & $4.46 \mathrm{E}+07$ & $2.2927 \mathrm{E}+07$ & $5.2800 \mathrm{E}+07$ \\
\hline
\end{tabular}




\section{Continued}

\begin{tabular}{|c|c|c|c|c|c|c|c|c|}
\hline 12 & 4 & $8.97 \mathrm{E}-02$ & $9.1622 \mathrm{E}-02$ & $8.920 \mathrm{E}-02$ & 0.1111 & $2.65 \mathrm{E}+09$ & $2.7023 \mathrm{E}+09$ & $2.6460 \mathrm{E}+09$ \\
\hline 12 & 5 & $1.59 \mathrm{E}-01$ & $1.6943 \mathrm{E}-01$ & $1.601 \mathrm{E}-01$ & 0.1542 & $4.37 \mathrm{E}+09$ & $4.6533 \mathrm{E}+09$ & $4.4367 \mathrm{E}+09$ \\
\hline 13 & 2 & $1.24 \mathrm{E}-02$ & $6.9183 \mathrm{E}-03$ & $1.300 \mathrm{E}-02$ & ---- & $1.31 \mathrm{E}+09$ & $7.3167 \mathrm{E}+08$ & $1.3680 \mathrm{E}+09$ \\
\hline 13 & 3 & $2.86 \mathrm{E}-04$ & $5.2481 \mathrm{E}-05$ & $4.000 \mathrm{E}-04$ & --- & $2.83 \mathrm{E}+07$ & $5.1933 \mathrm{E}+06$ & $3.5600 \mathrm{E}+07$ \\
\hline 13 & 4 & $1.96 \mathrm{E}-01$ & $2.0417 \mathrm{E}-01$ & $1.952 \mathrm{E}-01$ & 0.2017 & $8.02 \mathrm{E}+09$ & $8.3033 E+09$ & $7.9133 \mathrm{E}+09$ \\
\hline 13 & 5 & $9.70 \mathrm{E}-02$ & $1.0139 \mathrm{E}-01$ & $9.450 \mathrm{E}-02$ & 0.1224 & $3.74 \mathrm{E}+09$ & $3.8800 \mathrm{E}+09$ & $3.6167 \mathrm{E}+09$ \\
\hline 14 & 2 & $1.78 \mathrm{E}-03$ & $1.2823 \mathrm{E}-03$ & $1.800 \mathrm{E}-03$ & ---- & $1.16 \mathrm{E}+08$ & $8.3220 \mathrm{E}+07$ & $1.1690 \mathrm{E}+08$ \\
\hline 14 & 3 & $1.08 \mathrm{E}-03$ & $1.2853 \mathrm{E}-03$ & $1.000 \mathrm{E}-03$ & ---- & $6.57 \mathrm{E}+07$ & $7.8180 \mathrm{E}+07$ & $6.2340 \mathrm{E}+07$ \\
\hline 14 & 5 & $5.08 \mathrm{E}-01$ & $5.1642 \mathrm{E}-01$ & $5.074 \mathrm{E}-01$ & 0.5590 & $1.22 \mathrm{E}+10$ & $1.2278 \mathrm{E}+10$ & $1.2140 \mathrm{E}+10$ \\
\hline 15 & 3 & $5.19 \mathrm{E}-02$ & $3.7154 \mathrm{E}-02$ & $4.470 \mathrm{E}-02$ & ---- & $2.14 \mathrm{E}+10$ & $1.4450 \mathrm{E}+10$ & $1.7370 \mathrm{E}+10$ \\
\hline 15 & 5 & $1.15 \mathrm{E}-01$ & $1.0447 \mathrm{E}-01$ & $1.125 \mathrm{E}-01$ & 0.1002 & $2.19 \mathrm{E}+10$ & $1.8200 \mathrm{E}+10$ & $1.9640 \mathrm{E}+10$ \\
\hline 16 & 6 & $1.02 \mathrm{E}-01$ & $1.1015 \mathrm{E}-01$ & $9.950 \mathrm{E}-02$ & 0.1190 & $1.80 \mathrm{E}+10$ & $1.9500 \mathrm{E}+10$ & $1.7480 \mathrm{E}+10$ \\
\hline 16 & 13 & $8.34 \mathrm{E}-04$ & $7.3961 \mathrm{E}-04$ & $8.000 \mathrm{E}-04$ & ---- & $1.57 \mathrm{E}+07$ & $1.4110 \mathrm{E}+07$ & $1.5230 \mathrm{E}+07$ \\
\hline 17 & 1 & $9.08 \mathrm{E}-03$ & $1.7539 \mathrm{E}-02$ & $1.070 \mathrm{E}-02$ & ---- & $2.37 \mathrm{E}+11$ & $4.5833 \mathrm{E}+11$ & $2.7840 \mathrm{E}+11$ \\
\hline 17 & 6 & $2.41 \mathrm{E}-01$ & $2.5061 \mathrm{E}-01$ & $2.368 \mathrm{E}-01$ & 0.1374 & $1.50 \mathrm{E}+10$ & $1.5670 \mathrm{E}+10$ & $1.4670 \mathrm{E}+10$ \\
\hline 17 & 7 & $2.75 \mathrm{E}-02$ & $3.1477 \mathrm{E}-02$ & $2.580 \mathrm{E}-02$ & 0.0416 & $1.53 \mathrm{E}+09$ & $1.7543 \mathrm{E}+09$ & $1.4320 \mathrm{E}+09$ \\
\hline 17 & 9 & $1.03 \mathrm{E}-03$ & $2.2961 \mathrm{E}-03$ & $1.400 \mathrm{E}-03$ & 0.0578 & $4.56 \mathrm{E}+07$ & $1.0280 \mathrm{E}+08$ & $6.0967 \mathrm{E}+07$ \\
\hline 17 & 10 & $3.59 \mathrm{E}-02$ & $3.9902 \mathrm{E}-02$ & $3.570 \mathrm{E}-02$ & 0.0095 & $1.40 \mathrm{E}+09$ & $1.5723 \mathrm{E}+09$ & $1.4040 \mathrm{E}+09$ \\
\hline 17 & 11 & $3.27 \mathrm{E}-03$ & $5.1168 \mathrm{E}-03$ & $3.300 \mathrm{E}-03$ & 0.0551 & $7.69 \mathrm{E}+07$ & $1.2400 \mathrm{E}+08$ & $7.9167 \mathrm{E}+07$ \\
\hline 18 & 8 & $9.17 \mathrm{E}-01$ & $2.3227 \mathrm{E}-02$ & $1.880 \mathrm{E}-02$ & ---- & $1.54 \mathrm{E}+10$ & $7.1220 \mathrm{E}+08$ & $3.1800 \mathrm{E}+08$ \\
\hline 19 & 6 & $1.78 \mathrm{E}-01$ & ---- & 1.692E-01 & 0.1613 & $7.31 \mathrm{E}+09$ & --- & $3.8478 \mathrm{E}+09$ \\
\hline 19 & 7 & $7.27 \mathrm{E}-02$ & ---- & --- & 0.0483 & $2.69 \mathrm{E}+09$ & --- & --- \\
\hline 19 & 8 & $2.05 \mathrm{E}-02$ & $9.4842 \mathrm{E}-01$ & $9.122 \mathrm{E}-01$ & 0.0245 & $6.25 \mathrm{E}+08$ & $1.6189 \mathrm{E}+10$ & $2.7820 \mathrm{E}+10$ \\
\hline 19 & 9 & $5.28 \mathrm{E}-02$ & ---- & ---- & 0.0966 & $1.57 \mathrm{E}+09$ & ---- & ---- \\
\hline 19 & 10 & $1.94 \mathrm{E}-01$ & ---- & ---- & 0.2101 & $5.12 \mathrm{E}+09$ & ---- & ---- \\
\hline 19 & 12 & $2.59 \mathrm{E}-04$ & ---- & ---- & ---- & $2.58 \mathrm{E}+06$ & ---- & ---- \\
\hline 19 & 14 & $5.61 \mathrm{E}-04$ & --- & ---- & ---- & $3.02 \mathrm{E}+06$ & --- & ---- \\
\hline 20 & 7 & $6.62 \mathrm{E}-01$ & $6.8707 \mathrm{E}-01$ & $6.600 \mathrm{E}-01$ & 0.6821 & $1.76 \mathrm{E}+10$ & $1.8343 \mathrm{E}+10$ & $1.7643 \mathrm{E}+10$ \\
\hline 20 & 8 & $1.08 \mathrm{E}-01$ & $8.6896 \mathrm{E}-02$ & $1.058 \mathrm{E}-01$ & 0.1138 & $2.37 \mathrm{E}+09$ & $1.9229 \mathrm{E}+09$ & $2.3443 \mathrm{E}+09$ \\
\hline 20 & 10 & $9.78 \mathrm{E}-03$ & $2.7102 \mathrm{E}-02$ & $1.010 \mathrm{E}-02$ & 0.0087 & $1.86 \mathrm{E}+08$ & $5.2129 \mathrm{E}+08$ & $1.9514 \mathrm{E}+08$ \\
\hline 21 & 6 & $2.98 \mathrm{E}-02$ & $5.4325 \mathrm{E}-02$ & $3.670 \mathrm{E}-02$ & 0.0597 & $1.34 \mathrm{E}+09$ & $2.4340 \mathrm{E}+09$ & $1.6360 \mathrm{E}+09$ \\
\hline 21 & 7 & $1.75 \mathrm{E}-01$ & $2.0370 \mathrm{E}-01$ & ---- & 0.2015 & $7.08 \mathrm{E}+09$ & $8.2260 \mathrm{E}+09$ & $7.3560 \mathrm{E}+09$ \\
\hline 21 & 8 & $6.42 \mathrm{E}-03$ & $9.7051 \mathrm{E}-03$ & ---- & 0.0118 & $2.16 \mathrm{E}+08$ & $3.2740 \mathrm{E}+08$ & $2.4860 \mathrm{E}+08$ \\
\hline 21 & 9 & $3.47 \mathrm{E}-01$ & $3.0761 \mathrm{E}-01$ & ---- & 0.3133 & $1.14 \mathrm{E}+10$ & $1.0172 \mathrm{E}+10$ & $1.0940 \mathrm{E}+10$ \\
\hline 21 & 10 & $6.08 \mathrm{E}-03$ & $3.9355 \mathrm{E}-05$ & ---- & 0.0005 & $1.78 \mathrm{E}+08$ & $1.1614 \mathrm{E}+06$ & $9.4940 \mathrm{E}+07$ \\
\hline 22 & 7 & $3.17 \mathrm{E}-03$ & $2.3659 \mathrm{E}-04$ & ---- & 0.1574 & $9.73 \mathrm{E}+07$ & $7.2943 \mathrm{E}+06$ & $8.9543 \mathrm{E}+07$ \\
\hline 22 & 8 & $1.51 \mathrm{E}-01$ & $1.7219 \mathrm{E}-01$ & --- & 0.0045 & $3.87 \mathrm{E}+09$ & $4.4671 \mathrm{E}+09$ & $3.8886 \mathrm{E}+09$ \\
\hline 22 & 10 & $6.04 \mathrm{E}-01$ & $5.9429 \mathrm{E}-01$ & --- & 0.6387 & $1.36 \mathrm{E}+10$ & $1.3539 \mathrm{E}+10$ & $1.3590 \mathrm{E}+10$ \\
\hline 23 & 1 & $1.21 \mathrm{E}+00$ & $1.4060 \mathrm{E}+00$ & ---- & ---- & $3.23 \mathrm{E}+13$ & $3.7600 \mathrm{E}+13$ & $3.2120 \mathrm{E}+13$ \\
\hline 23 & 9 & $1.59 \mathrm{E}-01$ & $1.5560 \mathrm{E}-01$ & ---- & 0.0691 & $1.19 \mathrm{E}+10$ & $1.1437 \mathrm{E}+10$ & $1.1690 \mathrm{E}+10$ \\
\hline 23 & 11 & $1.54 \mathrm{E}-01$ & $1.5171 \mathrm{E}-01$ & ---- & 0.0934 & $7.27 \mathrm{E}+09$ & $7.0300 \mathrm{E}+09$ & $7.1367 \mathrm{E}+09$ \\
\hline 24 & 6 & $4.29 \mathrm{E}-04$ & $7.0469 \mathrm{E}-04$ & ---- & ---- & $3.81 \mathrm{E}+07$ & $6.1980 \mathrm{E}+07$ & $4.5200 \mathrm{E}+07$ \\
\hline 24 & 7 & $7.98 \mathrm{E}-04$ & $1.1561 \mathrm{E}-04$ & ---- & --- & $6.60 \mathrm{E}+07$ & $9.4500 \mathrm{E}+06$ & $6.1840 \mathrm{E}+07$ \\
\hline
\end{tabular}




\section{Continued}

\begin{tabular}{|c|c|c|c|c|c|c|c|c|}
\hline 24 & 10 & $3.70 \mathrm{E}-04$ & $7.4473 \mathrm{E}-04$ & ---- & ---- & $2.46 \mathrm{E}+07$ & $4.9180 \mathrm{E}+07$ & $3.8180 \mathrm{E}+07$ \\
\hline 24 & 12 & $4.79 \mathrm{E}-01$ & $4.7643 \mathrm{E}-01$ & ---- & 0.0191 & $1.83 \mathrm{E}+10$ & $1.7818 \mathrm{E}+10$ & $1.7880 \mathrm{E}+10$ \\
\hline 24 & 13 & $1.13 \mathrm{E}-02$ & $1.6943 \mathrm{E}-05$ & ---- & ---- & $3.34 \mathrm{E}+08$ & $4.9140 \mathrm{E}+05$ & $2.1240 \mathrm{E}+08$ \\
\hline 24 & 14 & $6.99 \mathrm{E}-02$ & $9.1201 \mathrm{E}-02$ & ---- & 0.0608 & $1.99 \mathrm{E}+09$ & $2.5640 \mathrm{E}+09$ & $2.0880 \mathrm{E}+09$ \\
\hline 25 & 6 & $9.36 \mathrm{E}-04$ & $6.1235 \mathrm{E}-04$ & ---- & ---- & $8.59 \mathrm{E}+07$ & $5.5860 \mathrm{E}+07$ & $6.6320 \mathrm{E}+07$ \\
\hline 25 & 7 & $2.24 \mathrm{E}-03$ & $3.6141 \mathrm{E}-03$ & ---- & ---- & $1.91 \mathrm{E}+08$ & $3.0660 \mathrm{E}+08$ & $2.0400 \mathrm{E}+08$ \\
\hline 25 & 8 & $3.72 \mathrm{E}-03$ & $1.3428 \mathrm{E}-03$ & ---- & ---- & $2.81 \mathrm{E}+08$ & $1.0084 \mathrm{E}+08$ & $3.4300 \mathrm{E}+08$ \\
\hline 25 & 9 & $1.27 \mathrm{E}-04$ & $4.6345 \mathrm{E}-06$ & ---- & ---- & $9.45 \mathrm{E}+06$ & $3.4380 \mathrm{E}+05$ & $1.2870 \mathrm{E}+07$ \\
\hline 25 & 10 & $8.28 \mathrm{E}-03$ & $6.5615 \mathrm{E}-03$ & ---- & ---- & $5.71 \mathrm{E}+08$ & $4.5180 \mathrm{E}+08$ & $6.4040 \mathrm{E}+08$ \\
\hline 25 & 12 & $2.48 \mathrm{E}-04$ & $9.8628 \mathrm{E}-03$ & ---- & 0.0028 & $9.91 \mathrm{E}+06$ & $3.8980 \mathrm{E}+08$ & $1.7430 \mathrm{E}+06$ \\
\hline 25 & 13 & $4.55 \mathrm{E}-01$ & $4.7863 \mathrm{E}-01$ & ---- & 0.4467 & $1.41 \mathrm{E}+10$ & $1.4806 \mathrm{E}+10$ & $1.4020 \mathrm{E}+10$ \\
\hline 25 & 14 & $5.15 \mathrm{E}-02$ & $3.3651 \mathrm{E}-02$ & ---- & 0.0653 & $1.55 \mathrm{E}+09$ & $1.0066 \mathrm{E}+09$ & $1.3530 \mathrm{E}+09$ \\
\hline 26 & 7 & $2.06 \mathrm{E}-04$ & $4.4157 \mathrm{E}-05$ & ---- & ---- & $1.29 \mathrm{E}+07$ & $2.7300 \mathrm{E}+06$ & $1.5157 \mathrm{E}+07$ \\
\hline 26 & 8 & $2.27 \mathrm{E}-03$ & $2.3174 \mathrm{E}-03$ & ---- & ---- & $1.26 \mathrm{E}+08$ & $1.2674 \mathrm{E}+08$ & $1.3200 \mathrm{E}+08$ \\
\hline 26 & 10 & $1.87 \mathrm{E}-04$ & $7.6384 \mathrm{E}-04$ & ---- & ---- & $9.45 \mathrm{E}+06$ & $3.8286 \mathrm{E}+07$ & $1.0320 \mathrm{E}+07$ \\
\hline 26 & 14 & $7.32 \mathrm{E}-01$ & $7.4131 \mathrm{E}-01$ & ---- & 0.7396 & $1.63 \mathrm{E}+10$ & $1.6329 \mathrm{E}+10$ & $1.6200 \mathrm{E}+10$ \\
\hline 27 & 1 & $2.14 \mathrm{E}+00$ & $1.9861 \mathrm{E}+00$ & ---- & ---- & $5.97 \mathrm{E}+13$ & $5.5400 \mathrm{E}+13$ & $5.9600 \mathrm{E}+13$ \\
\hline 27 & 12 & $1.11 \mathrm{E}-01$ & $1.1041 \mathrm{E}-01$ & ---- & 0.0948 & $9.62 \mathrm{E}+09$ & $9.0600 \mathrm{E}+09$ & $9.1033 \mathrm{E}+09$ \\
\hline 27 & 15 & $1.44 \mathrm{E}-01$ & $1.5524 \mathrm{E}-01$ & ---- & 0.1571 & $6.28 \mathrm{E}+09$ & $6.9133 \mathrm{E}+09$ & $6.8567 \mathrm{E}+09$ \\
\hline 28 & 2 & $3.12 \mathrm{E}-01$ & ---- & --- & ---- & $1.70 \mathrm{E}+11$ & ---- & $9.3233 \mathrm{E}+10$ \\
\hline 28 & 3 & $7.53 \mathrm{E}-02$ & ---- & ---- & ---- & $3.97 \mathrm{E}+10$ & ---- & $2.1890 \mathrm{E}+10$ \\
\hline 28 & 4 & $5.48 \mathrm{E}-02$ & ---- & ---- & --- & $2.07 \mathrm{E}+10$ & ---- & $1.0920 \mathrm{E}+10$ \\
\hline 28 & 5 & $9.63 \mathrm{E}-02$ & ---- & ---- & ---- & $3.56 \mathrm{E}+10$ & ---- & $1.8670 \mathrm{E}+10$ \\
\hline 29 & 3 & $7.57 \mathrm{E}-02$ & ---- & ---- & ---- & $1.32 \mathrm{E}+11$ & ---- & $6.7650 \mathrm{E}+10$ \\
\hline 29 & 5 & $3.91 \mathrm{E}-02$ & ---- & ---- & ---- & $4.86 \mathrm{E}+10$ & ---- & $2.1740 \mathrm{E}+10$ \\
\hline 29 & 23 & $2.05 \mathrm{E}-03$ & ---- & ---- & ---- & $5.43 \mathrm{E}+08$ & ---- & $1.3400 \mathrm{E}+08$ \\
\hline 29 & 27 & $3.36 \mathrm{E}-03$ & ---- & ---- & 0.0003 & $3.38 \mathrm{E}+08$ & ---- & $2.6180 \mathrm{E}+07$ \\
\hline 30 & 6 & $7.81 \mathrm{E}-02$ & ---- & ---- & ---- & $1.30 \mathrm{E}+11$ & ---- & $6.2310 \mathrm{E}+10$ \\
\hline 30 & 9 & $3.01 \mathrm{E}-02$ & ---- & ---- & ---- & $4.51 \mathrm{E}+10$ & ---- & $2.1320 \mathrm{E}+10$ \\
\hline 30 & 12 & $5.20 \mathrm{E}-02$ & ---- & ---- & ---- & $5.86 \mathrm{E}+10$ & ---- & $2.4570 \mathrm{E}+10$ \\
\hline 30 & 13 & $1.28 \mathrm{E}-03$ & ---- & ---- & ---- & $1.31 \mathrm{E}+09$ & ---- & $9.2910 \mathrm{E}+08$ \\
\hline 30 & 28 & $9.37 \mathrm{E}-02$ & ---- & ---- & 0.0866 & $8.21 \mathrm{E}+09$ & ---- & $4.9130 \mathrm{E}+09$ \\
\hline 31 & 1 & $6.68 \mathrm{E}-02$ & ---- & ---- & ---- & $2.10 \mathrm{E}+12$ & ---- & $1.6030 \mathrm{E}+12$ \\
\hline 31 & 6 & $4.63 \mathrm{E}-02$ & ---- & ---- & ---- & $2.60 \mathrm{E}+10$ & ---- & $1.2140 \mathrm{E}+10$ \\
\hline 31 & 7 & $2.89 \mathrm{E}-01$ & ---- & $2.054 \mathrm{E}-01$ & ---- & $1.56 \mathrm{E}+11$ & ---- & $7.3700 \mathrm{E}+10$ \\
\hline 31 & 9 & $7.46 \mathrm{E}-04$ & ---- & $1.200 \mathrm{E}-03$ & ---- & $3.75 \mathrm{E}+08$ & ---- & $3.9933 \mathrm{E}+08$ \\
\hline 31 & 10 & $4.70 \mathrm{E}-03$ & ---- & $4.100 \mathrm{E}-03$ & ---- & $2.28 \mathrm{E}+09$ & ---- & $1.2790 \mathrm{E}+09$ \\
\hline 31 & 11 & $1.73 \mathrm{E}-02$ & ---- & $1.140 \mathrm{E}-02$ & ---- & $7.37 \mathrm{E}+09$ & ---- & $3.0550 \mathrm{E}+09$ \\
\hline 31 & 12 & $9.38 \mathrm{E}-02$ & ---- & $6.860 \mathrm{E}-02$ & ---- & $3.56 \mathrm{E}+10$ & ---- & $1.5760 \mathrm{E}+10$ \\
\hline 31 & 13 & $4.43 \mathrm{E}-03$ & --- & $3.700 \mathrm{E}-03$ & --- & $1.52 \mathrm{E}+09$ & --- & $7.5467 \mathrm{E}+08$ \\
\hline 31 & 14 & $2.32 \mathrm{E}-03$ & ---- & $1.200 \mathrm{E}-03$ & --- & $7.86 \mathrm{E}+08$ & ---- & $2.2760 \mathrm{E}+08$ \\
\hline 31 & 15 & $3.62 \mathrm{E}-02$ & ---- & $3.250 \mathrm{E}-02$ & ---- & $1.02 \mathrm{E}+10$ & ---- & $5.3233 \mathrm{E}+09$ \\
\hline 31 & 28 & $2.35 \mathrm{E}-01$ & ---- & $1.990 \mathrm{E}-01$ & 0.2284 & $7.12 \mathrm{E}+09$ & ---- & $4.3367 \mathrm{E}+09$ \\
\hline
\end{tabular}




\section{Continued}

\begin{tabular}{|c|c|c|c|c|c|c|c|c|}
\hline 31 & 29 & $3.84 \mathrm{E}-02$ & --- & $3.270 \mathrm{E}-02$ & --- & $7.34 \mathrm{E}+08$ & ---- & $4.5700 \mathrm{E}+08$ \\
\hline 32 & 6 & $5.93 \mathrm{E}-03$ & ---- & $2.600 \mathrm{E}-03$ & ---- & $2.14 \mathrm{E}+09$ & ---- & $6.4740 \mathrm{E}+08$ \\
\hline 32 & 7 & $1.59 \mathrm{E}-03$ & ---- & $9.000 \mathrm{E}-04$ & ---- & $5.54 \mathrm{E}+08$ & ---- & $2.1140 \mathrm{E}+08$ \\
\hline 32 & 8 & $4.35 \mathrm{E}-01$ & --- & $3.034 \mathrm{E}-01$ & --- & $1.42 \mathrm{E}+11$ & --- & $6.6100 \mathrm{E}+10$ \\
\hline 32 & 9 & $2.05 \mathrm{E}-02$ & ---- & $1.370 \mathrm{E}-02$ & --- & $6.65 \mathrm{E}+09$ & ---- & $2.9480 \mathrm{E}+09$ \\
\hline 32 & 10 & $1.28 \mathrm{E}-01$ & ---- & $9.580 \mathrm{E}-02$ & ---- & $4.02 \mathrm{E}+10$ & ---- & $1.9850 \mathrm{E}+10$ \\
\hline 32 & 12 & $9.76 \mathrm{E}-04$ & ---- & $8.000 \mathrm{E}-04$ & ---- & $2.41 \mathrm{E}+08$ & ---- & $1.1380 \mathrm{E}+08$ \\
\hline 32 & 13 & $8.13 \mathrm{E}-02$ & ---- & $5.510 \mathrm{E}-02$ & --- & $1.82 \mathrm{E}+10$ & ---- & $7.4940 \mathrm{E}+09$ \\
\hline 32 & 14 & $1.20 \mathrm{E}-01$ & ---- & $8.710 \mathrm{E}-02$ & ---- & $2.65 \mathrm{E}+10$ & ---- & $1.1660 \mathrm{E}+10$ \\
\hline 32 & 28 & $5.61 \mathrm{E}-01$ & ---- & $4.884 \mathrm{E}-01$ & ---- & $1.35 \mathrm{E}+10$ & ---- & $8.8360 \mathrm{E}+09$ \\
\hline 33 & 1 & $2.94 \mathrm{E}-01$ & --- & $2.689 \mathrm{E}-01$ & --- & $9.37 \mathrm{E}+12$ & --- & $8.1633 E+12$ \\
\hline 33 & 6 & $6.25 \mathrm{E}-03$ & ---- & $4.300 \mathrm{E}-03$ & ---- & $3.85 \mathrm{E}+09$ & ---- & $1.7920 \mathrm{E}+09$ \\
\hline 33 & 7 & $6.11 \mathrm{E}-03$ & ---- & $4.400 \mathrm{E}-03$ & ---- & $3.63 \mathrm{E}+09$ & ---- & $1.7370 \mathrm{E}+09$ \\
\hline 33 & 9 & $1.18 \mathrm{E}-01$ & ---- & $8.710 \mathrm{E}-02$ & ---- & $6.55 \mathrm{E}+10$ & ---- & $3.2250 \mathrm{E}+10$ \\
\hline 33 & 10 & $1.36 \mathrm{E}-01$ & ---- & $9.750 \mathrm{E}-02$ & --- & $7.27 \mathrm{E}+10$ & ---- & $3.4667 \mathrm{E}+10$ \\
\hline 33 & 11 & $3.92 \mathrm{E}-02$ & ---- & $3.350 \mathrm{E}-02$ & --- & $1.86 \mathrm{E}+10$ & ---- & $1.0200 \mathrm{E}+10$ \\
\hline 33 & 12 & $8.19 \mathrm{E}-03$ & ---- & $7.400 \mathrm{E}-03$ & ---- & $3.48 \mathrm{E}+09$ & ---- & $1.9470 \mathrm{E}+09$ \\
\hline 33 & 13 & $3.96 \mathrm{E}-02$ & ---- & $3.140 \mathrm{E}-02$ & ---- & $1.53 \mathrm{E}+10$ & ---- & $7.3833 \mathrm{E}+09$ \\
\hline 33 & 14 & $1.19 \mathrm{E}-01$ & ---- & $8.090 \mathrm{E}-02$ & ---- & $4.54 \mathrm{E}+10$ & ---- & $1.8700 \mathrm{E}+10$ \\
\hline 33 & 15 & $4.86 \mathrm{E}-02$ & ---- & $3.990 \mathrm{E}-02$ & ---- & $1.55 \mathrm{E}+10$ & ---- & $7.6933 \mathrm{E}+09$ \\
\hline 33 & 28 & $6.38 \mathrm{E}-02$ & ---- & $5.630 \mathrm{E}-02$ & 0.0697 & $2.80 \mathrm{E}+09$ & ---- & $1.8650 \mathrm{E}+09$ \\
\hline 33 & 29 & $2.21 \mathrm{E}-01$ & --- & $1.892 \mathrm{E}-01$ & 0.2430 & $6.69 \mathrm{E}+09$ & --- & $4.4000 \mathrm{E}+09$ \\
\hline 34 & 16 & $2.63 \mathrm{E}-02$ & ---- & $1.660 \mathrm{E}-02$ & ---- & $1.46 \mathrm{E}+10$ & ---- & $5.9533 \mathrm{E}+09$ \\
\hline 34 & 17 & $6.04 \mathrm{E}-02$ & ---- & $3.970 \mathrm{E}-02$ & ---- & $3.30 \mathrm{E}+10$ & ---- & $1.3880 \mathrm{E}+10$ \\
\hline 34 & 19 & $3.26 \mathrm{E}-02$ & --- & --- & --- & $1.72 \mathrm{E}+10$ & --- & --- \\
\hline 34 & 21 & $1.13 \mathrm{E}-01$ & --- & $8.290 \mathrm{E}-02$ & --- & $5.80 \mathrm{E}+10$ & --- & $2.6690 \mathrm{E}+10$ \\
\hline 34 & 23 & $4.12 \mathrm{E}-02$ & --- & $2.970 \mathrm{E}-02$ & --- & $1.87 \mathrm{E}+10$ & ---- & $8.2633 \mathrm{E}+09$ \\
\hline 34 & 24 & $1.09 \mathrm{E}-01$ & ---- & $7.540 \mathrm{E}-02$ & ---- & $3.97 \mathrm{E}+10$ & ---- & $1.5690 \mathrm{E}+10$ \\
\hline 34 & 25 & $8.82 \mathrm{E}-04$ & ---- & $2.000 \mathrm{E}-04$ & ---- & $3.15 \mathrm{E}+08$ & ---- & $5.5600 \mathrm{E}+07$ \\
\hline 34 & 27 & $1.63 \mathrm{E}-02$ & ---- & $1.250 \mathrm{E}-02$ & ---- & $5.13 \mathrm{E}+09$ & ---- & $2.1360 \mathrm{E}+09$ \\
\hline 34 & 30 & $1.98 \mathrm{E}-01$ & ---- & $1.875 \mathrm{E}-01$ & 0.2028 & $1.16 \mathrm{E}+10$ & ---- & $1.0040 \mathrm{E}+10$ \\
\hline 34 & 31 & $1.19 \mathrm{E}-01$ & --- & $1.107 \mathrm{E}-01$ & 0.1190 & $6.80 \mathrm{E}+09$ & --- & $5.7400 \mathrm{E}+09$ \\
\hline 34 & 32 & $8.58 \mathrm{E}-03$ & ---- & $8.000 \mathrm{E}-03$ & ---- & $3.91 \mathrm{E}+08$ & ---- & $3.2560 \mathrm{E}+08$ \\
\hline 34 & 33 & $2.28 \mathrm{E}-02$ & ---- & $2.230 \mathrm{E}-02$ & 0.0207 & $9.49 \mathrm{E}+08$ & ---- & $8.3367 \mathrm{E}+08$ \\
\hline 35 & 17 & $2.53 \mathrm{E}-02$ & ---- & $1.570 \mathrm{E}-02$ & ---- & $8.32 \mathrm{E}+09$ & ---- & $3.3160 \mathrm{E}+09$ \\
\hline 35 & 19 & $9.06 \mathrm{E}-02$ & --- & ---- & ---- & $2.88 \mathrm{E}+10$ & --- & --- \\
\hline 35 & 20 & $3.05 \mathrm{E}-01$ & --- & $2.106 \mathrm{E}-01$ & ---- & $9.68 \mathrm{E}+10$ & --- & $4.2360 \mathrm{E}+10$ \\
\hline 35 & 21 & $5.55 \mathrm{E}-03$ & ---- & ---- & --- & $1.71 \mathrm{E}+09$ & ---- & $9.6780 \mathrm{E}+08$ \\
\hline 35 & 22 & $3.89 \mathrm{E}-02$ & ---- & ---- & ---- & $1.17 \mathrm{E}+10$ & ---- & $5.5680 \mathrm{E}+09$ \\
\hline 35 & 23 & $3.11 \mathrm{E}-02$ & ---- & ---- & ---- & $8.53 \mathrm{E}+09$ & ---- & $3.4440 \mathrm{E}+09$ \\
\hline 35 & 24 & $7.59 \mathrm{E}-02$ & ---- & ---- & ---- & $1.67 \mathrm{E}+10$ & ---- & $6.0480 \mathrm{E}+09$ \\
\hline 35 & 25 & $3.18 \mathrm{E}-02$ & ---- & ---- & ---- & $6.85 \mathrm{E}+09$ & ---- & $3.3620 \mathrm{E}+09$ \\
\hline 35 & 26 & $4.46 \mathrm{E}-02$ & ---- & ---- & ---- & $9.47 \mathrm{E}+09$ & ---- & $3.8180 \mathrm{E}+09$ \\
\hline
\end{tabular}




\begin{tabular}{|c|c|c|c|c|c|c|c|c|}
\hline \multicolumn{9}{|c|}{ Continued } \\
\hline 35 & 27 & $2.23 \mathrm{E}-02$ & ---- & --- & --- & $4.24 \mathrm{E}+09$ & ---- & $1.1170 \mathrm{E}+09$ \\
\hline 35 & 31 & $3.98 \mathrm{E}-01$ & ---- & --- & 0.4009 & $1.39 \mathrm{E}+10$ & ---- & $1.1860 \mathrm{E}+10$ \\
\hline 35 & 32 & $1.30 \mathrm{E}-01$ & ---- & --- & ---- & $3.60 \mathrm{E}+09$ & ---- & $3.0320 \mathrm{E}+09$ \\
\hline 35 & 33 & $4.04 \mathrm{E}-02$ & ---- & --- & 0.0403 & $1.02 \mathrm{E}+09$ & ---- & $9.4340 \mathrm{E}+08$ \\
\hline 36 & 18 & $5.29 \mathrm{E}-01$ & ---- & --- & ---- & $1.22 \mathrm{E}+11$ & ---- & $2.4014 \mathrm{E}+09$ \\
\hline 36 & 19 & $2.52 \mathrm{E}-02$ & ---- & ---- & ---- & $5.79 \mathrm{E}+09$ & ---- & $5.4543 \mathrm{E}+10$ \\
\hline 36 & 20 & $5.20 \mathrm{E}-04$ & ---- & --- & ---- & $1.19 \mathrm{E}+08$ & ---- & $7.0343 \mathrm{E}+07$ \\
\hline 36 & 21 & $1.27 \mathrm{E}-02$ & ---- & --- & ---- & $2.82 \mathrm{E}+09$ & ---- & $1.0570 \mathrm{E}+09$ \\
\hline 36 & 22 & $1.65 \mathrm{E}-01$ & ---- & ---- & ---- & $3.59 \mathrm{E}+10$ & ---- & $1.5700 \mathrm{E}+10$ \\
\hline 36 & 24 & $1.53 \mathrm{E}-03$ & ---- & --- & ---- & $2.44 \mathrm{E}+08$ & ---- & $7.1843 \mathrm{E}+07$ \\
\hline 36 & 25 & $8.08 \mathrm{E}-02$ & --- & --- & ---- & $1.26 \mathrm{E}+10$ & --- & $3.9943 \mathrm{E}+09$ \\
\hline 36 & 26 & $1.33 \mathrm{E}-01$ & ---- & --- & ---- & $2.04 \mathrm{E}+10$ & ---- & $7.9014 \mathrm{E}+09$ \\
\hline 36 & 32 & $7.51 \mathrm{E}-01$ & ---- & ---- & ---- & $1.54 \mathrm{E}+10$ & ---- & $1.3070 \mathrm{E}+10$ \\
\hline 37 & 3 & $6.24 \mathrm{E}-04$ & ---- & --- & ---- & $5.14 \mathrm{E}+08$ & ---- & $2.8880 \mathrm{E}+08$ \\
\hline 37 & 5 & $5.56 \mathrm{E}-04$ & ---- & ---- & ---- & $3.70 \mathrm{E}+08$ & ---- & $2.3160 \mathrm{E}+08$ \\
\hline 37 & 17 & $1.26 \mathrm{E}-03$ & ---- & ---- & ---- & $4.51 \mathrm{E}+08$ & ---- & $2.2120 \mathrm{E}+08$ \\
\hline 37 & 19 & $2.48 \mathrm{E}-02$ & ---- & --- & ---- & $8.57 \mathrm{E}+09$ & ---- & ---- \\
\hline 37 & 20 & $1.04 \mathrm{E}-01$ & ---- & ---- & ---- & $3.58 \mathrm{E}+10$ & ---- & $1.7050 \mathrm{E}+10$ \\
\hline 37 & 21 & $1.20 \mathrm{E}-01$ & ---- & ---- & ---- & $4.03 \mathrm{E}+10$ & ---- & $1.7640 \mathrm{E}+10$ \\
\hline 37 & 22 & $1.48 \mathrm{E}-01$ & ---- & --- & ---- & $4.87 \mathrm{E}+10$ & ---- & $2.1580 \mathrm{E}+10$ \\
\hline 37 & 23 & $3.73 \mathrm{E}-02$ & ---- & ---- & ---- & $1.12 \mathrm{E}+10$ & ---- & $4.3680 \mathrm{E}+09$ \\
\hline 37 & 24 & $3.98 \mathrm{E}-02$ & ---- & --- & ---- & $9.64 \mathrm{E}+09$ & ---- & $4.2460 \mathrm{E}+09$ \\
\hline 37 & 25 & $4.45 \mathrm{E}-02$ & ---- & --- & ---- & $1.06 \mathrm{E}+10$ & ---- & $3.8760 \mathrm{E}+09$ \\
\hline 37 & 26 & $1.42 \mathrm{E}-01$ & ---- & --- & ---- & $3.34 \mathrm{E}+10$ & ---- & $1.3290 \mathrm{E}+10$ \\
\hline 37 & 27 & $3.24 \mathrm{E}-02$ & ---- & --- & --- & $6.84 \mathrm{E}+09$ & ---- & $1.6950 \mathrm{E}+09$ \\
\hline 37 & 31 & $8.53 \mathrm{E}-02$ & ---- & ---- & 0.0855 & $3.78 \mathrm{E}+09$ & ---- & $3.5220 \mathrm{E}+09$ \\
\hline 37 & 32 & $1.24 \mathrm{E}-03$ & ---- & ---- & 0.0019 & $4.49 \mathrm{E}+07$ & ---- & $2.9640 \mathrm{E}+07$ \\
\hline 37 & 33 & $5.14 \mathrm{E}-01$ & ---- & ---- & 0.5069 & $1.72 \mathrm{E}+10$ & ---- & $1.4760 \mathrm{E}+10$ \\
\hline 38 & 6 & $1.56 \mathrm{E}-01$ & $1.6368 \mathrm{E}-01$ & --- & ---- & $2.09 \mathrm{E}+11$ & $2.2080 \mathrm{E}+11$ & ---- \\
\hline 38 & 7 & $1.23 \mathrm{E}-01$ & $1.3274 \mathrm{E}-01$ & --- & --- & $1.62 \mathrm{E}+11$ & $1.7580 \mathrm{E}+11$ & --- \\
\hline 38 & 8 & $3.78 \mathrm{E}-01$ & $3.8548 \mathrm{E}-01$ & --- & ---- & $4.83 \mathrm{E}+11$ & $4.9520 \mathrm{E}+11$ & ---- \\
\hline 38 & 10 & $1.55 \mathrm{E}-01$ & $1.4859 \mathrm{E}-01$ & ---- & ---- & $1.94 \mathrm{E}+11$ & $1.8694 \mathrm{E}+11$ & ---- \\
\hline 38 & 12 & $1.32 \mathrm{E}-04$ & $1.7701 \mathrm{E}-04$ & ---- & --- & $1.47 \mathrm{E}+08$ & $1.9826 \mathrm{E}+08$ & --- \\
\hline 38 & 13 & $3.36 \mathrm{E}-03$ & $3.0130 \mathrm{E}-03$ & ---- & ---- & $3.57 \mathrm{E}+09$ & $3.2240 \mathrm{E}+09$ & ---- \\
\hline 38 & 14 & $9.20 \mathrm{E}-04$ & $5.7810 \mathrm{E}-04$ & ---- & ---- & $9.74 \mathrm{E}+08$ & $6.1580 \mathrm{E}+08$ & ---- \\
\hline
\end{tabular}

i and $\mathbf{j}$ : The lower (i) and upper (j) levels of a transition as defined in Table 4. gf (nm) (FAC): Weighted oscillator strengths from the FAC code (our calculation). gf (nm) (COWAN): Weighted oscillator strengths from the COWAN code (also calculation). gf (nm) (CIV3): Weighted oscillator

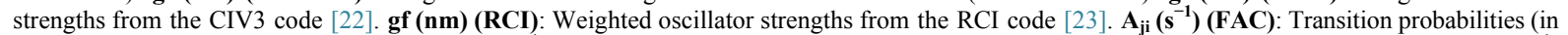
$\mathrm{nm}$ ) from the FAC code (our calculation). $\mathbf{A}_{\mathbf{j i}}\left(\mathbf{s}^{-1}\right)$ (COWAN): Transition probabilities (in nm) from the COWAN code (also calculation). $\mathbf{A}_{\mathbf{j i}}\left(\mathbf{s}^{-1}\right)$ (CIV3): Transition probabilities (in $\mathrm{nm}$ ) from the CIV3 code [22].

\section{Gain Coefficient Computations}

The possibility of laser emission from plasma of Ge XXIII ion via electron collisional pumping, in the XUV and soft X-ray spectral regions is investigated at different plasma temperatures and plasma electron densities. The reduced population densities are calculated by solving the coupled rate equations [13] [14]. 


$$
N_{j}\left[\sum_{i<j} A_{j i}+N_{e}\left(\sum_{i<j} C_{j i}^{d}+\sum_{i>j} C_{j i}^{e}\right)\right]=N_{e}\left(\sum_{i<j} N_{i} C_{i j}^{e}+\sum_{i>j} N_{i} C_{i j}^{d}\right)+\sum_{i>j} N_{i} A_{i j}
$$

where $N_{j}$ and $N_{i}$ is the fractional population of level $j$ and $i$ respectively, $N_{e}$ is the electron density, $A_{j i}$ is the Einstein coefficient for spontaneous radiative decay from $j$ to $i$; and $C_{i j}^{e}$ and $C_{j i}^{d}$ represent the rate coefficient for collisional excitation and de-excitation respectively. The actual population density $N_{J}$ of the $j^{\text {th }}$ level can be calculated from the equation of identity [15] [16].

$$
C_{j i}^{d}=C_{i j}^{e}\left[\frac{g_{i}}{g_{j}}\right] \exp \left[\frac{\Delta E_{j i}}{k T_{e}}\right]
$$

where $g_{i}$ and $g_{j}$ are the statistical weights of the lower and upper levels, respectively.

The electron impact excitation rates usually are expressed via the effective collision strengths $\gamma_{j i}$ as

$$
C_{j i}^{d}=\frac{8.6287 \times 10^{-6}}{g_{j} T_{e}^{1 / 2}} \gamma_{i j}
$$

where the values of $\gamma_{j i}$ and $A_{j i}$ are obtained by [17].

The actual population density $N_{J}$ of the $j^{\text {th }}$ level is obtained from the following identity [17],

$$
N_{J}=N_{j} \times N_{i}
$$

where $N_{I}$ is the quantity of ions which reached to the ionization stage $I$ [17],

$$
N_{I}=f_{I} N_{e} / Z_{\text {avg }}
$$

where $N_{e}$ is the electron density, $Z_{\text {avg }}$ is the average degree of ionization and $f_{I}$ is the fractional abundance of the ionization states which can be calculated from the relation [17]. Since the populations calculated from Equation (1) are normalized such that,

$$
\sum_{J=1}^{241} \frac{N_{J}}{N_{I}}=1
$$

where 241 is the number of all the levels of the ion under consideration, the quantity actually obtained from Equation (1) is the fractional population $N_{J} / N_{I}$. After the calculation of levels population, the quantities $N_{j} / g_{j}$ and $N_{i} / g_{i}$ can be calculated. Application of electron collisional pumping, the collision in the laser ion plasma will transfer the pumped quanta to other levels, and will result in population inversions between the upper and lower levels. Once a population inversion has been ensured a positive gain through $F>0 \quad[18]$ is obtained.

$$
F=\frac{g_{j}}{N_{j}}\left[\frac{N_{j}}{g_{j}}-\frac{N_{i}}{g_{i}}\right]
$$

where $N_{j} / g_{j}$ and $N_{i} / g_{i}$ are the reduced populations of the upper level and lower level respectively. Equation (7) has been used to calculate the gain coefficient $(\alpha)$ for Doppler broadening of the various transitions in the Ge XXIII ion.

$$
\alpha_{j i}=\frac{\lambda_{i j}^{3}}{8 \pi}\left[\frac{M}{2 \pi K T_{i}}\right]^{1 / 2} A_{j i} N_{j} F
$$

where $M$ is the ion mass $\lambda_{i j}$ is the transition wavelength in cm (see Table 3), $T_{i}$ is the ion temperature in $K$ and $j, I$ represents the upper and lower transition levels respectively.

As seen from Equation (8), the gain coefficient is expressed in terms of the upper state density $\left(N_{j}\right)$. This quantity $N_{j}$ depends on how the upper state is populated, as well as on the density of the initial source state. The source state is often the ground state for the particular ion.

\section{Results and Discussions}

\subsection{Energy Levels}

The energy level values obtained using the fully relativistic flexible atomic code (FAC) for the $1 \mathrm{~s}^{2} 2 \mathrm{~s}^{2} 2 \mathrm{p}^{5} \mathrm{nl}, 1 \mathrm{~s}^{2}$ 
Table 3. Comparison between some wavelengths $(\lambda)$ for E1 transitions of Ge XXIII (in $\mathrm{nm}$ ).

\begin{tabular}{|c|c|c|c|c|c|c|c|}
\hline $\mathrm{j}$ & $\mathrm{i}$ & $\lambda(\mathrm{nm})(\mathrm{FAC})$ & $\lambda(\mathrm{nm})(\mathrm{COWAN})$ & $\lambda(\mathrm{nm})(\mathrm{CIV} 3)$ & $\lambda(\mathrm{nm})(\mathrm{MCDF})$ & $\lambda(\mathrm{nm})(\mathrm{RCI})$ & $\lambda(\mathrm{nm})(\mathrm{Exp})$. \\
\hline 3 & 1 & 1.00291911 & 1.0009 & 1.001 & ---- & ---- & ---- \\
\hline 5 & 1 & 0.978298138 & 0.9763 & 0.976 & ---- & ---- & ---- \\
\hline 6 & 2 & 29.06054601 & 29.0804 & 29.1 & --- & ---- & ---- \\
\hline 7 & 2 & 26.82759761 & 26.7880 & 26.85 & ---- & ---- & ---- \\
\hline 7 & 3 & 28.60854399 & 28.5017 & 28.48 & 28.644 & 28.432 & 28.628 \\
\hline 8 & 2 & 23.76385089 & 23.7695 & 23.82 & 23.760 & 24.192 & 23.767 \\
\hline 9 & 2 & 23.38618832 & 23.5053 & 23.51 & 23.395 & 24.151 & 23.385 \\
\hline 9 & 3 & 24.72809857 & 24.8145 & 24.75 & 24.727 & 25.422 & 24.735 \\
\hline 10 & 2 & 22.00458606 & 22.0738 & 22.16 & ---- & ---- & ---- \\
\hline 10 & 3 & 23.18861213 & 23.2245 & 23.26 & 23.229 & 24.033 & 23.224 \\
\hline 10 & 5 & 55.46243419 & 56.1774 & 56.06 & ---- & ---- & ---- \\
\hline 11 & 3 & 19.06599826 & 19.1780 & 19.09 & ---- & ---- & ---- \\
\hline 11 & 5 & 36.55639248 & 37.1945 & 36.72 & --- & ---- & ---- \\
\hline 12 & 2 & 15.97797365 & 15.9610 & 15.98 & ---- & ---- & ---- \\
\hline 12 & 3 & 16.59318561 & 16.5540 & 16.54 & ---- & ---- & ---- \\
\hline 12 & 4 & 27.43670009 & 27.4431 & 27.39 & ---- & ---- & ---- \\
\hline 12 & 5 & 28.43226568 & 28.4487 & 28.32 & 28.475 & 28.318 & 28.509 \\
\hline 13 & 2 & 14.48681287 & 14.4972 & 14.54 & ---- & ---- & ---- \\
\hline 13 & 3 & 14.99074178 & 14.9848 & 15 & ---- & ---- & ---- \\
\hline 13 & 4 & 23.31563846 & 23.3836 & 23.42 & 23.319 & 23.390 & 23.314 \\
\hline 13 & 5 & 24.03069931 & 24.1098 & 24.1 & 24.044 & 23.900 & 24.049 \\
\hline 14 & 2 & 14.32320141 & 14.3395 & 14.36 & ---- & ---- & ---- \\
\hline 14 & 3 & 14.81561859 & 14.8164 & 14.81 & ---- & ---- & ---- \\
\hline 14 & 5 & 23.58382453 & 23.6768 & 23.61 & 23.627 & 23.407 & 23.625 \\
\hline 15 & 3 & 12.74125872 & 13.0918 & 13.1 & ---- & ---- & ---- \\
\hline 15 & 5 & 18.72982602 & 19.5593 & 19.55 & 19.544 & 19.399 & 19.619 \\
\hline 16 & 6 & 19.4465226 & 19.4149 & 19.49 & 19.399 & 19.701 & 19.460 \\
\hline 16 & 13 & 59.50360748 & 59.1173 & 59.16 & ---- & ---- & ---- \\
\hline 17 & 1 & 0.924189369 & 0.9223 & 0.922 & ---- & ---- & ---- \\
\hline 17 & 6 & 18.92164195 & 18.8596 & 18.94 & 18.878 & 19.045 & 18.940 \\
\hline 17 & 7 & 20.00584033 & 19.9678 & 20.03 & ---- & ---- & ---- \\
\hline 17 & 9 & 22.47182417 & 22.2880 & 22.41 & --- & ---- & ---- \\
\hline 17 & 10 & 23.91464589 & 23.7483 & 23.78 & ---- & ---- & ---- \\
\hline 17 & 11 & 30.77812808 & 30.2817 & 30.63 & ---- & ---- & ---- \\
\hline 18 & 8 & 20.99113251 & 20.8451 & 20.93 & ---- & ---- & ---- \\
\hline 19 & 6 & 18.02121903 & ---- & 18.05 & ---- & ---- & ---- \\
\hline 19 & 7 & 19.00201092 & ---- & ---- & 18.895 & 18.877 & 18.961 \\
\hline 19 & 8 & 20.91160561 & 20.8281 & 20.91 & ---- & ---- & ---- \\
\hline 19 & 9 & 21.21305773 & ---- & ---- & ---- & ---- & ---- \\
\hline 19 & 10 & 22.49415888 & ---- & ---- & ---- & ---- & ---- \\
\hline 19 & 12 & 36.61005733 & ---- & ---- & ---- & ---- & ---- \\
\hline 19 & 14 & 49.79022451 & ---- & ---- & --- & ---- & ---- \\
\hline
\end{tabular}




\section{Continued}

\begin{tabular}{|c|c|c|c|c|c|c|c|}
\hline 20 & 7 & 18.94688793 & 18.9013 & 18.88 & 18.814 & 18.874 & 18.880 \\
\hline 20 & 8 & 20.84486629 & 20.7616 & 20.74 & ---- & ---- & ---- \\
\hline 20 & 10 & 22.4169586 & 22.2548 & 22.18 & ---- & ---- & ---- \\
\hline 21 & 6 & 17.27924434 & 17.2601 & 17.3 & ---- & ---- & ---- \\
\hline 21 & 7 & 18.17892 & 18.1837 & 18.2 & ---- & ---- & ---- \\
\hline 21 & 8 & 19.9190906 & 19.8990 & 19.92 & 19.814 & 19.669 & 19.873 \\
\hline 21 & 9 & 20.1924195 & 20.0880 & 20.14 & ---- & ---- & ---- \\
\hline 21 & 10 & 21.3498462 & 21.2667 & 21.25 & ---- & ---- & ---- \\
\hline 22 & 7 & 17.6454186 & 17.5769 & 17.67 & ---- & ---- & ---- \\
\hline 22 & 8 & 19.2803566 & 19.1747 & 19.29 & 19.192 & 18.936 & 19.259 \\
\hline 22 & 10 & 20.617748 & 20.4414 & 20.53 & 20.475 & 19.815 & 20.539 \\
\hline 23 & 1 & 0.91269295 & 0.9117 & 0.911 & ---- & ---- & ---- \\
\hline 23 & 9 & 17.2029364 & 17.3848 & 17.21 & ---- & ---- & ---- \\
\hline 23 & 11 & 21.6825457 & 21.8925 & 21.67 & ---- & ---- & ---- \\
\hline 24 & 6 & 12.2585873 & 12.3094 & 12.28 & ---- & ---- & ---- \\
\hline 24 & 7 & 12.704653 & 12.7720 & 12.73 & ---- & ---- & ---- \\
\hline 24 & 10 & 14.1760934 & 14.2199 & 14.14 & ---- & ---- & ---- \\
\hline 24 & 12 & 18.7265184 & 18.8773 & 18.79 & ---- & ---- & ---- \\
\hline 24 & 13 & 21.295593 & 21.4373 & 21.26 & ---- & ---- & ---- \\
\hline 24 & 14 & 21.6592895 & 21.7917 & 21.65 & ---- & ---- & ---- \\
\hline 25 & 6 & 12.0678472 & 12.0887 & 12.1 & ---- & ---- & ---- \\
\hline 25 & 7 & 12.4998937 & 12.5346 & 12.54 & ---- & ---- & ---- \\
\hline 25 & 8 & 13.2987553 & 13.3265 & 13.33 & ---- & ---- & ---- \\
\hline 25 & 9 & 13.4200377 & 13.4110 & 13.43 & ---- & ---- & ---- \\
\hline 25 & 10 & 13.921634 & 13.9263 & 13.91 & ---- & ---- & ---- \\
\hline 25 & 12 & 18.285025 & 18.3634 & 18.38 & ---- & ---- & ---- \\
\hline 25 & 13 & 20.7264947 & 20.7769 & 20.74 & ---- & ---- & ---- \\
\hline 25 & 14 & 21.0708515 & 21.1096 & 21.11 & ---- & ---- & ---- \\
\hline 26 & 7 & 12.3577809 & 12.4198 & 12.38 & ---- & ---- & ---- \\
\hline 26 & 8 & 13.138017 & 13.1968 & 13.15 & ---- & ---- & ---- \\
\hline 26 & 10 & 13.7455841 & 13.7847 & 13.71 & ---- & ---- & ---- \\
\hline 26 & 14 & 20.6701616 & 20.7860 & 20.65 & 20.519 & 20.644 & 20.583 \\
\hline 27 & 1 & 0.89303659 & 0.8927 & 0.892 & ---- & ---- & ---- \\
\hline 27 & 12 & 16.0204352 & 16.4624 & 16.1 & ---- & ---- & ---- \\
\hline 27 & 15 & 22.6240255 & 22.3370 & 21.61 & ---- & ---- & --- \\
\hline 28 & 2 & 6.39977902 & ---- & 7.52 & ---- & ---- & ---- \\
\hline 28 & 3 & 6.49625131 & ---- & 7.64 & ---- & ---- & ---- \\
\hline 28 & 4 & 7.68540454 & ---- & 9.36 & ---- & ---- & ---- \\
\hline 28 & 5 & 7.76153141 & ---- & 9.46 & ---- & ---- & ---- \\
\hline 29 & 3 & 6.19256802 & ---- & 7.29 & ---- & ---- & ---- \\
\hline 29 & 5 & 7.33194183 & ---- & 8.93 & ---- & ---- & ---- \\
\hline 29 & 23 & 15.8945958 & ---- & 25.93 & ---- & ---- & ---- \\
\hline 29 & 27 & 25.774576 & ---- & 68.98 & ---- & ---- & ---- \\
\hline
\end{tabular}




\section{Continued}

\begin{tabular}{|c|c|c|c|c|c|c|c|}
\hline 30 & 6 & 6.32686538 & --- & 7.74 & --- & --- & --- \\
\hline 30 & 9 & 6.67972533 & --- & 8.27 & --- & --- & --- \\
\hline 30 & 12 & 7.69935951 & --- & 9.91 & --- & --- & --- \\
\hline 30 & 13 & 8.10118282 & --- & 10.56 & --- & --- & --- \\
\hline 30 & 28 & 27.6154168 & --- & 32.75 & ---- & --- & --- \\
\hline 31 & 1 & 0.84183128 & --- & 0.861 & ---- & --- & --- \\
\hline 31 & 6 & 6.30092271 & --- & 7.7 & --- & --- & --- \\
\hline 31 & 7 & 6.416725578 & --- & 7.87 & --- & --- & --- \\
\hline 31 & 9 & 6.650814849 & --- & 8.21 & ---- & --- & --- \\
\hline 31 & 10 & 6.77173044 & --- & 8.39 & --- & --- & --- \\
\hline 31 & 11 & 7.228153142 & --- & 9.11 & --- & --- & --- \\
\hline 31 & 12 & 7.660979329 & --- & 9.83 & ---- & --- & --- \\
\hline 31 & 13 & 8.058697858 & --- & 10.47 & --- & --- & --- \\
\hline 31 & 14 & 8.110232992 & --- & 10.57 & --- & --- & --- \\
\hline 31 & 15 & 8.903756621 & --- & 11.65 & ---- & --- & --- \\
\hline 31 & 28 & 27.1279177 & --- & 31.94 & ---- & --- & --- \\
\hline 31 & 29 & 34.1140454 & --- & 39.92 & ---- & ---- & ---- \\
\hline 32 & 6 & 6.089149806 & --- & 7.38 & ---- & --- & --- \\
\hline 32 & 7 & 6.197229399 & --- & 7.54 & ---- & --- & ---- \\
\hline 32 & 8 & 6.387459838 & --- & 7.82 & --- & --- & --- \\
\hline 32 & 9 & 6.415305514 & --- & 7.86 & --- & ---- & --- \\
\hline 32 & 10 & 6.527736953 & --- & 8.02 & ---- & --- & --- \\
\hline 32 & 12 & 7.35016756 & --- & 9.33 & ---- & --- & ---- \\
\hline 32 & 13 & 7.715504567 & --- & 9.9 & --- & --- & --- \\
\hline 32 & 14 & 7.762726026 & ---- & 9.98 & ---- & ---- & --- \\
\hline 32 & 28 & 23.59488012 & ---- & 27.15 & ---- & ---- & --- \\
\hline 33 & 1 & 0.836515264 & --- & 0.856 & ---- & --- & --- \\
\hline 33 & 6 & 6.014819126 & --- & 7.29 & --- & --- & --- \\
\hline 33 & 7 & 6.120253309 & --- & 7.44 & --- & ---- & --- \\
\hline 33 & 9 & 6.33285591 & --- & 7.75 & --- & ---- & --- \\
\hline 33 & 10 & 6.442391307 & --- & 7.91 & ---- & --- & --- \\
\hline 33 & 11 & 6.854144356 & --- & 8.54 & --- & ---- & --- \\
\hline 33 & 12 & 7.242135475 & --- & 9.18 & ---- & --- & --- \\
\hline 33 & 13 & 7.596553105 & ---- & 9.73 & --- & ---- & ---- \\
\hline 33 & 14 & 7.642330131 & --- & 9.81 & ---- & --- & ---- \\
\hline 33 & 15 & 8.342972988 & --- & 10.74 & --- & ---- & --- \\
\hline 33 & 28 & 22.51667288 & --- & 25.91 & ---- & --- & --- \\
\hline 33 & 29 & 27.12777534 & --- & 30.91 & --- & ---- & --- \\
\hline 34 & 16 & 6.32759139 & --- & 7.88 & ---- & ---- & --- \\
\hline 34 & 17 & 6.385224484 & ---- & 7.97 & --- & ---- & --- \\
\hline 34 & 19 & 6.494731238 & --- & --- & --- & --- & --- \\
\hline 34 & 21 & 6.596819889 & --- & 8.31 & --- & --- & ---- \\
\hline 34 & 23 & 6.993883236 & --- & 8.94 & ---- & ---- & ---- \\
\hline
\end{tabular}




\section{Continued}

\begin{tabular}{|c|c|c|c|c|c|c|c|}
\hline 34 & 24 & 7.819488144 & --- & 10.34 & ---- & ---- & ---- \\
\hline 34 & 25 & 7.89912776 & --- & 10.46 & ---- & ---- & ---- \\
\hline 34 & 27 & 8.412867128 & --- & 11.38 & --- & --- & --- \\
\hline 34 & 30 & 19.45339221 & --- & 20.38 & --- & 19.340 & 19.209 \\
\hline 34 & 31 & 19.70280794 & --- & 20.71 & --- & --- & --- \\
\hline 34 & 32 & 22.10702133 & --- & 23.38 & ---- & ---- & ---- \\
\hline 34 & 33 & 23.14544859 & --- & 24.39 & ---- & ---- & ---- \\
\hline 35 & 17 & 6.370709831 & --- & 7.95 & --- & ---- & ---- \\
\hline 35 & 19 & 6.479715048 & --- & ---- & ---- & ---- & --- \\
\hline 35 & 20 & 6.486151251 & --- & 8.144 & --- & ---- & ---- \\
\hline 35 & 21 & 6.58132848 & --- & 8.28 & --- & --- & ---- \\
\hline 35 & 22 & 6.65416425 & --- & 8.393 & --- & ---- & --- \\
\hline 35 & 23 & 6.97647331 & --- & 8.9 & ---- & ---- & ---- \\
\hline 35 & 24 & 7.79773163 & --- & 10.29 & ---- & ---- & ---- \\
\hline 35 & 25 & 7.87692645 & --- & 10.42 & ---- & ---- & --- \\
\hline 35 & 26 & 7.93442318 & --- & 10.53 & --- & ---- & ---- \\
\hline 35 & 27 & 8.38768868 & --- & 11.33 & ---- & ---- & ---- \\
\hline 35 & 31 & 19.5652593 & --- & 20.52 & --- & 19.544 & 19.414 \\
\hline 35 & 32 & 21.9340035 & --- & 23.15 & ---- & ---- & --- \\
\hline 35 & 33 & 22.9558645 & --- & 24.14 & ---- & ---- & ---- \\
\hline 36 & 18 & 6.44169557 & --- & 8.07 & ---- & ---- & --- \\
\hline 36 & 19 & 6.44922283 & --- & 8.07 & ---- & ---- & ---- \\
\hline 36 & 20 & 6.45559522 & --- & 8.096 & ---- & ---- & ---- \\
\hline 36 & 21 & 6.54987129 & --- & 8.23 & ---- & ---- & ---- \\
\hline 36 & 22 & 6.62200863 & ---- & 8.342 & ---- & ---- & ---- \\
\hline 36 & 24 & 7.75361057 & --- & 10.21 & ---- & ---- & ---- \\
\hline 36 & 25 & 7.83190723 & --- & 10.34 & --- & ---- & ---- \\
\hline 36 & 26 & 7.88874624 & --- & 10.45 & --- & --- & ---- \\
\hline 36 & 32 & 21.5884556 & --- & 22.76 & --- & --- & ---- \\
\hline 37 & 3 & 4.02622122 & --- & 4.6 & --- & --- & ---- \\
\hline 37 & 5 & 4.47873387 & --- & 5.21 & --- & --- & --- \\
\hline 37 & 17 & 6.11875924 & --- & 7.57 & --- & --- & ---- \\
\hline 37 & 19 & 6.21924501 & --- & --- & --- & --- & --- \\
\hline 37 & 20 & 6.22517394 & --- & 7.745 & --- & ---- & ---- \\
\hline 37 & 21 & 6.3127944 & --- & 7.87 & --- & --- & ---- \\
\hline 37 & 22 & 6.37977744 & --- & 7.969 & --- & --- & ---- \\
\hline 37 & 23 & 6.6754629 & --- & 8.43 & --- & --- & ---- \\
\hline 37 & 24 & 7.42357701 & --- & 9.66 & --- & ---- & ---- \\
\hline 37 & 25 & 7.4953238 & --- & 9.77 & --- & --- & --- \\
\hline 37 & 26 & 7.54736614 & --- & 9.87 & --- & ---- & ---- \\
\hline 37 & 27 & 7.95634814 & --- & 10.57 & --- & --- & --- \\
\hline 37 & 31 & 17.3688062 & --- & 18.16 & --- & ---- & --- \\
\hline 37 & 32 & 19.2105275 & --- & 20.19 & --- & ---- & ---- \\
\hline
\end{tabular}




\section{Continued}

\begin{tabular}{|c|c|c|c|c|c|c|c|}
\hline 37 & 33 & 19.9898721 & ---- & 20.94 & --- & 19.959 & 19.925 \\
\hline 38 & 6 & 3.15614026 & 3.1459 & ---- & --- & ---- & ---- \\
\hline 38 & 7 & 3.18493053 & 3.1753 & ---- & ---- & ---- & ---- \\
\hline 38 & 8 & 3.23443596 & 3.2238 & ---- & ---- & ---- & ---- \\
\hline 38 & 10 & 3.27002 & 3.2577 & ---- & --- & ---- & ---- \\
\hline 38 & 12 & 3.46419347 & 3.4529 & ---- & --- & ---- & ---- \\
\hline 38 & 13 & 3.5432683 & 3.5300 & ---- & ---- & ---- & ---- \\
\hline 38 & 14 & 3.55319554 & 3.5395 & ---- & ---- & ---- & ---- \\
\hline
\end{tabular}

$\mathbf{i}$ and $\mathbf{j}$ : The lower (i) and upper (j) levels of a transition as defined in Table 4. $\lambda(\mathbf{n m})$ (FAC): Transition wavelengths (in nm) from the FAC code (our calculation). $\lambda(\mathbf{n m})(\mathbf{C O W A N})$ : Transition wavelengths (in $\mathrm{nm}$ ) from the COWAN code (also calculation). $\lambda(\mathbf{n m})(\mathbf{C I V} 3)$ : Transition wavelengths (in nm) from the CIV3 code [22]. $\lambda(\mathbf{n m})$ (MCDF): Transition wavelengths (in nm) from the MCDF code [23]. $\lambda$ (nm) (RCI): Transition wavelengths (in $\mathrm{nm}$ ) from the RCI code [23]. $\lambda$ (nm) (Exp.): Transition wavelengths (in $\mathrm{nm}$ ) calculated experimentally [23].

$2 \mathrm{~s}^{1} 2 \mathrm{p}^{6} \mathrm{nl}(\mathrm{n}=3,4,5,6 ; 1=\mathrm{s}, \mathrm{p}, \mathrm{d}, \mathrm{f}, \mathrm{g}$ and $\mathrm{h})$ configurations in Ne-like Ge ions are presented in Table 1 . The main components of the computed Eigen vectors are also given in these tables in the jj-coupling schemes. For Ge XXIII, the agreement between FAC, MCDF, and other theoretical and experimental energies [19]-[23] with the values available at the National Institute of Standards and Technology (NIST) [24] and is within values less than $0.5 \%$ for a majority of levels.

\subsection{Radiative Decay Rates}

The oscillator strengths $\left(f_{i j}\right)$ and radiative rates $\left(A_{i j}\right)$ (in $\mathrm{s}^{-1}$ ) for a transition $i \rightarrow j$ are related by the following expression

$$
f_{i j}=\frac{m c}{8 \pi^{2} e^{2}} \lambda_{i j}^{2} \frac{\omega_{j}}{\omega_{i}} A_{j i}=1.49 \times 10^{-16} \lambda_{i j}^{2} \frac{\omega_{j}}{\omega_{i}} A_{j i}
$$

where $m$ and $e$ are the electron mass and charge, respectively, $c$ is the velocity of light, $\lambda_{i j}$ is the transition wavelength in $\AA$ and $\omega_{i}$ and $\omega_{j}$ are the statistical weights of the lower $i$ and upper $j$ levels, respectively. Similarly, the oscillator strength $\left(f_{i j}\right)$

(Dimensionless) and the line strength $S$ (in atomic units, 1 a. u. $=6.46 \times 10^{-36} \mathrm{~cm}^{2} \cdot$ eus ${ }^{2}$ ) are related by the following standard equations. For the electric dipole (E1) transitions,

$$
A_{j i}=\frac{2.0261 \times 10^{18}}{\omega_{j} \lambda_{i j}^{3}} S^{E 1} \text { and } f_{i j}=\frac{303.75}{\omega_{j} \lambda_{i j}^{3}} S^{E 1}
$$

The wavelengths, transition probabilities, and weighted oscillator strengths, for E1 transitions calculated using the (FAC) are reported in Table 2 and Table 3 for Ge XXIII. We present a comparison between our results and other theoretical transition probabilities for allowed E1 transitions for Ge XXIII in tables respectively. It shows that our results are in a good agreement with the other theoretical and experimental results [22] [23]. Table 2 shows a comparison between the FAC, MCDF, RCI, and other theoretical weighted oscillator strength [22] [23] values for some transitions among the levels of the $1 s^{2} 2 s^{2} 2 p^{5} n l, 1 s^{2} 2 s^{1} 2 p^{6} n l(n=3,4,5,6 ; 1=s, p, d, f$, g and h) configurations for Ge XXIII. Generally, there are no discrepancies between our FAC, COWAN, CIV3, MCDF, RCI, and experimental calculations and the agreement is within $\leq 20 \%$ for strong transitions.

\subsection{Level Population}

The reduced population densities are calculated for 241 finestructure levels arising from $1 s^{2} 2 s^{2} 2 p^{6} n l, 1 s^{2} 2 s^{2}$ $2 \mathrm{p}^{5} \mathrm{nl}, 1 \mathrm{~s}^{2} 2 \mathrm{~s}^{1} 2 \mathrm{p}^{6} \mathrm{nl}(\mathrm{n}=3,4,5,6 ; 1=\mathrm{s}, \mathrm{p}, \mathrm{d}, \mathrm{f}, \mathrm{g}$ and $\mathrm{h})$ configurations that emit radiation in the XUV and soft $\mathrm{X}$-ray spectral regions. The calculations were performed by solving the coupled rate Equation (1) simultaneously using MATLAB R2013acomputer program. The present calculations for the reduced populations as a function of electron densities are plotted in Figure 1 to Figure 4 for levels $(5,15,33,37)$ of the configurations $\left[\left(2 \mathrm{p}_{122} 3 \mathrm{~s}_{122}\right)_{1,}\left(2 \mathrm{p}_{122} 3 \mathrm{p}_{112}\right)_{0},\left(2 \mathrm{~s}_{112} 3 \mathrm{p}_{122}\right)_{1,}\left(2 \mathrm{~s}_{112} 3 \mathrm{~d}_{512}\right)_{2}\right]$ at different plasma temperatures $(650,850,1050,1250,1450$, 


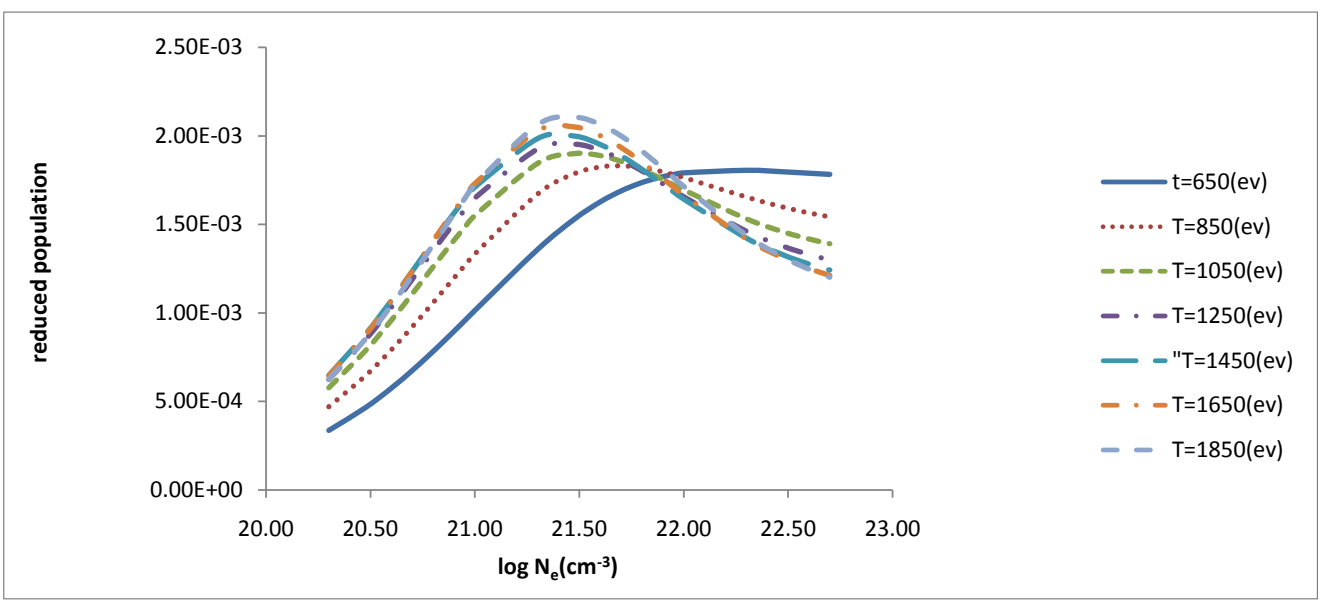

Figure 1. Reduced population of level $\left(2 \mathrm{p}_{12} 3 \mathrm{~s}_{12}\right)_{1}$ for Ge XXIII after electron collisional pumping as a function of the electron density at temperatures $(650,850,1050,1250,1450,1650$, and 1850) $\mathrm{eV}$.

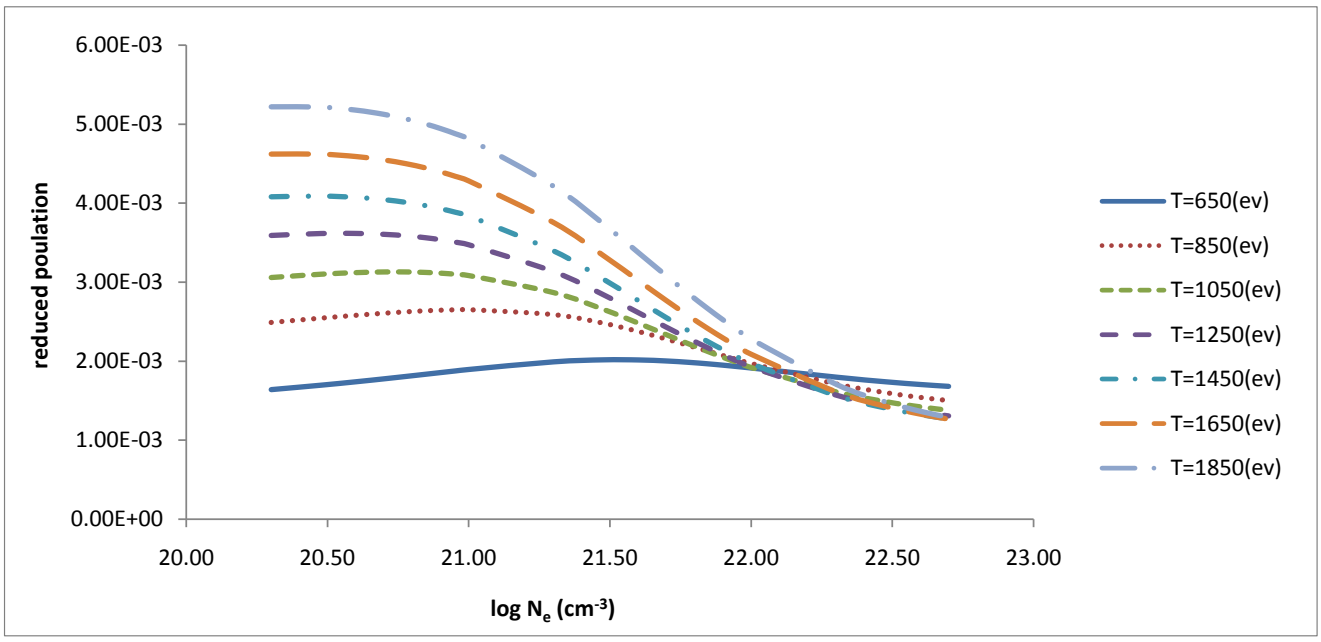

Figure 2. Reduced population of level $\left(2 \mathrm{p}_{122} 3 \mathrm{p}_{112}\right)_{0}$ for Ge XXIII after electron collisional pumping as a function of the electron density at temperatures $(650,850,1050,1250,1450,1650$, and 1850) $\mathrm{eV}$.

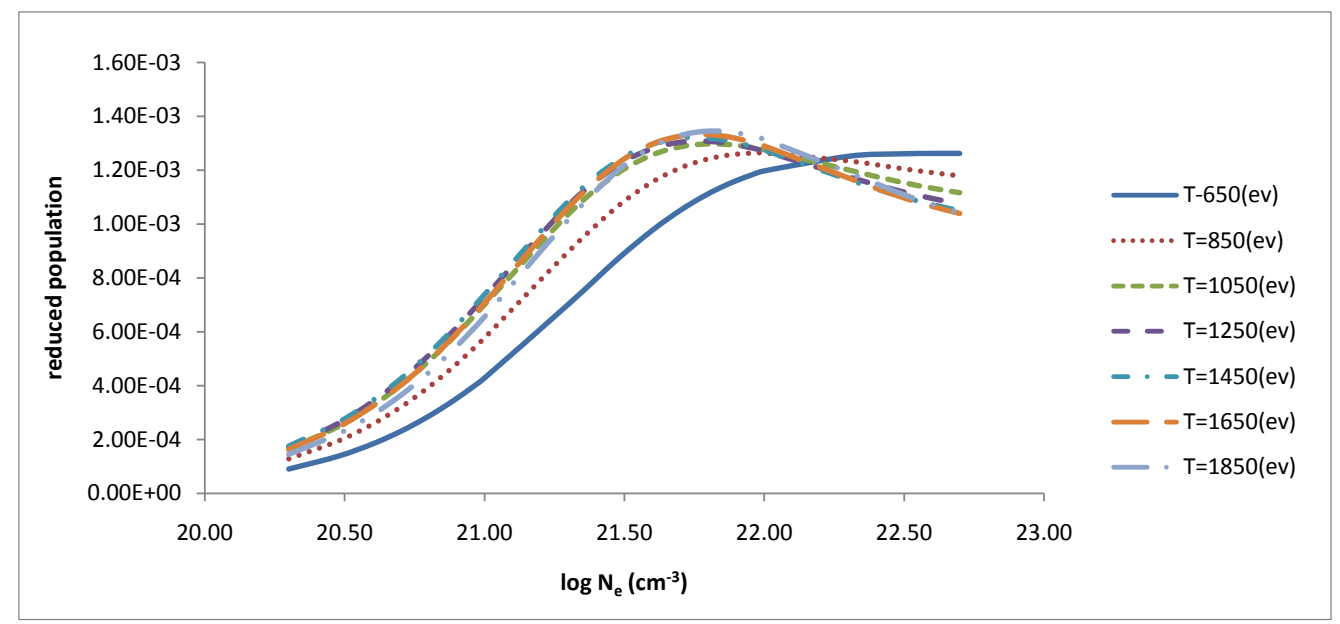

Figure 3. Reduced population of level $\left(2 \mathrm{~s}_{12} 3 \mathrm{p}_{122}\right)_{1}$ for Ge XXIII after electron collisional pumping as a function of the electron density at temperatures $(650,850,1050,1250,1450,1650$, and 1850) eV. 


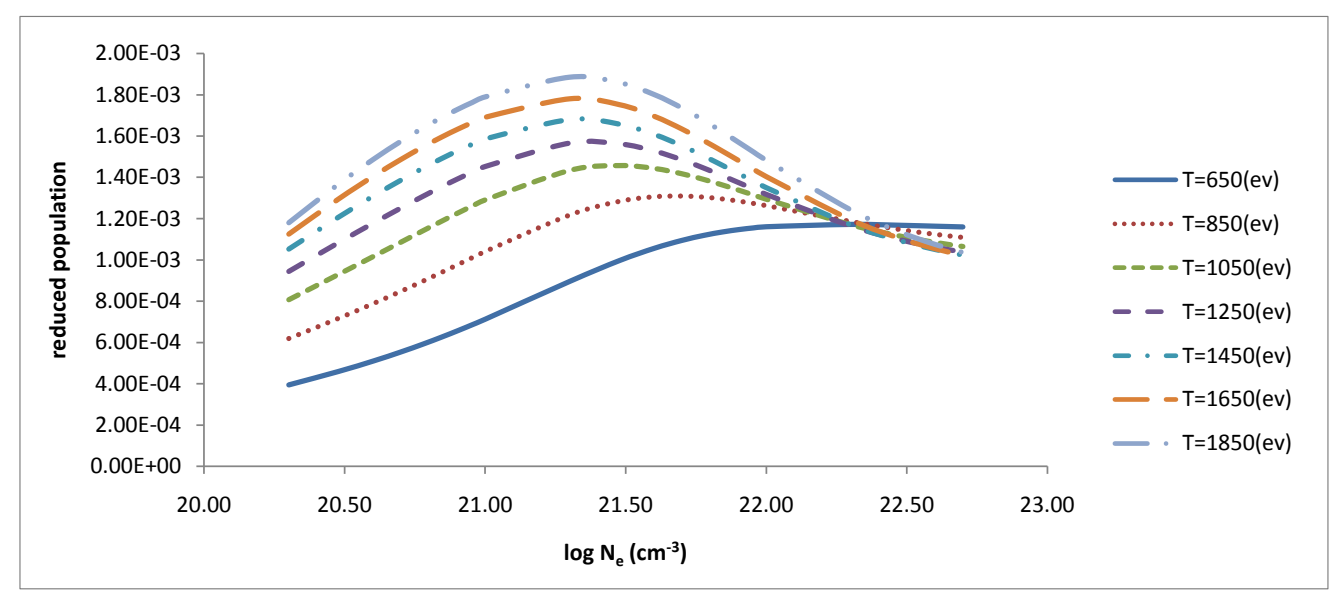

Figure 4. Reduced population of level $\left(2 \mathrm{~s}_{112} 3 \mathrm{~d}_{512}\right)_{2}$ for Ge XXIII after electron collisional pumping as a function of the electron density at temperatures $(650,850,1050,1250,1450,1650$, and 1850) eV.

1650 , and 1850) eV for Ge XXIII ion. In the calculation we took into account spontaneous radiative decay rate and electron collisional processes between all levels under the study. The behavior of level populations of the various ions can be explained as follows: in general at low electron densities the reduced population density is proportional to the electron density, where excitation to an excited state is followed immediately by radiative decay, and collisional mixing of excited levels can be ignored. This result is in agreement with that of Feldman et al. [19]-[21]. At high electron densities $\left(\mathrm{Ne}>5 \times 10^{22}\right)$, the radiative decay to all the levels will be negligible compared to collisional depopulations and all the level populations become independent of the electron density and are approximately equal (see Figures 1 to 4 ).

\subsection{Radiative Lifetime}

The lifetimes are determined almost entirely from the allowed and the strong inter combination transitions. The radiative lifetime $\tau_{j}$ of an excited atomic state $j$, is related to the atomic transition probability $A_{j i}$ by:

$$
\tau_{j}=\frac{1}{\sum_{i} A_{j i}}
$$

where the sum is extended over all the lower states which can be reached from the upper state by radiative decay.

Table 4 contains the present results of radiative lifetime for the upper and lower laser levels for the Ge XXIII.

\subsection{Inversion Factor}

As we mentioned before, laser amplification will occur only if there is population inversion, or in other words, for positive inversion factor $\mathrm{F}>0$. However, large amplification, the gain exceeds all losses and ensures laser emission. In order to work in the XUV and X-ray spectral regions, we have selected transitions between any two levels producing photons with wavelengths between 12 and $52 \mathrm{~nm}$. The electron density at which the population reaches corona equilibrium approximately equals to $\mathrm{A} / \mathrm{D}$, where $\mathrm{A}$ is the radiative decay rate and $\mathrm{D}$ is the collisional de-excitation rate [19] [21]. The population in version is largest where the electron collisions de-excitation rate for the upper level is comparable to the radiative decay rate for this level.

\subsection{Gain Coefficient}

As a result of population inversion there will be positive gain in laser medium. Equation (8) has been used to calculate gain coefficient for the Doppler broadening of various transitions in the Ge XXIII ion. Our results for the maximum gain coefficient in $\mathrm{cm}^{-1}$ for those transitions having appositive inversion factor $\mathrm{F}>0$ in the case of Ge XXIII ion at different temperatures are calculated (see Table 4) and plotted against electron density in Figure 5 and Figure 6. The figures show that the population inversions occur for several transitions in the Ge 


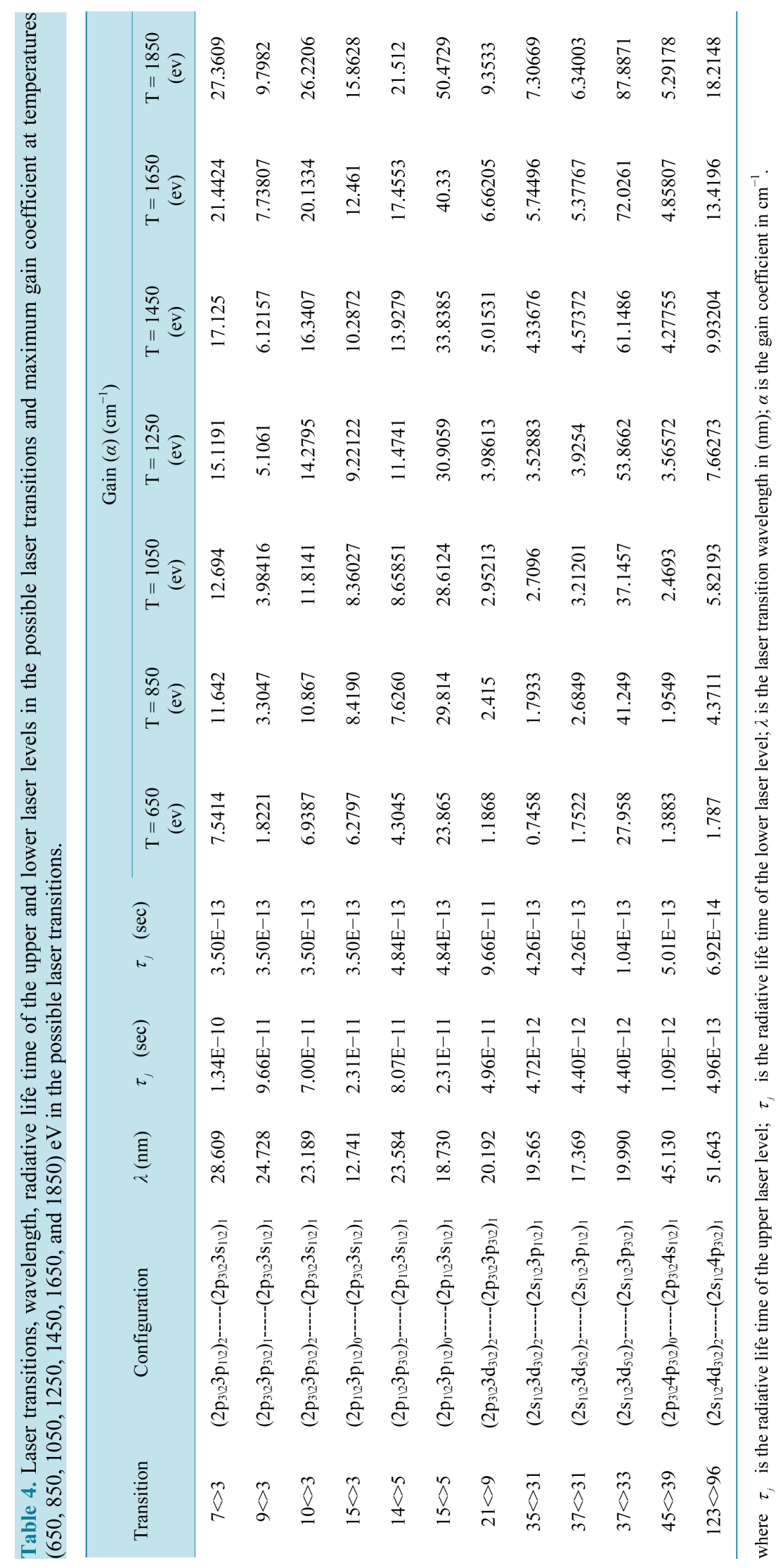




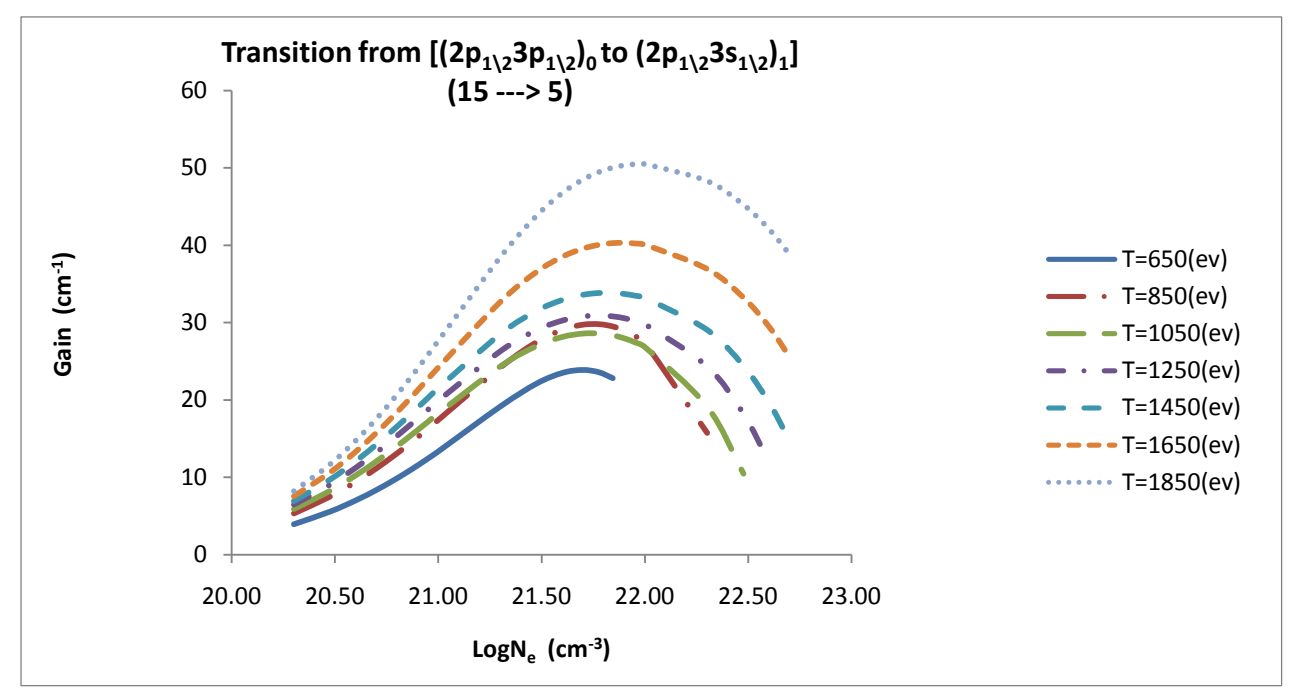

Figure 5. Gain coefficient against electron density $(\mathrm{Ne})$ at different temperatures $(650,850,1050$, $1250,1450,1650,1850) \mathrm{eV}$.

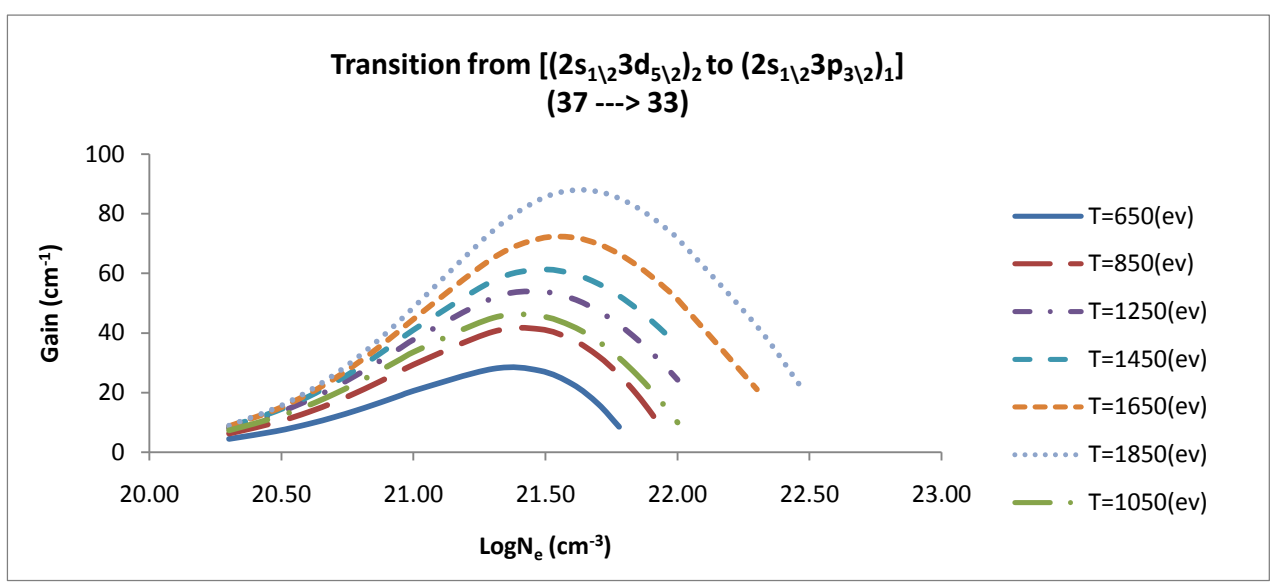

Figure 6. Gain coefficient against electron density $(\mathrm{Ne})$ at different temperatures $(650,850,1050$, $1250,1450,1650,1850) \mathrm{eV}$.

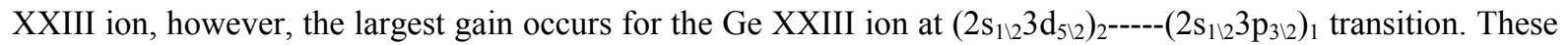
short wavelength laser transitions can be produced using plasmas created by optical lasers as the lasing medium. For Ge XXIII ion the rates for electron collisional excitation from the $1 s^{2} 2 s^{2} 2 p^{6}$ ground state to the $1 s^{2} 2 s^{1} 2 p^{6} 3 d$ configuration are greater than the rates for excitation from the ground state to the $1 s^{2} 2 s^{1} 2 p^{6} 3 p$ state. For electron densities and electron temperatures that are typical of laboratory high-density plasma sources, such as laser produced plasmas, it is possible to create a quasi-stationary population inversion between the $2 s^{1} 2 p^{6} 3 d$ and $2 s^{1}$ $2 p^{6} 3 p$ states in Ge XXIII ion. Our calculations have shown that under favorable conditions large laser gains for this transition in the XUV and soft X-ray regions of the spectrum can be achieved in the Ge XXIII. The gain calculations were performed at electron temperatures equal to $(650,850,1050,1250,1450,1650$, and 1850) eV at different electron densities. It is obvious that the gain increases with the temperature. We also find that some wavelengths for the lasing transitions in Table 4 are much closed to the values of it in the ref. [23] which give us the accurate wavelength calculated experimentally, although we Ignores some physical processes in the our rate equation.

\section{Conclusions}

The analysis of how the electron collisional pumping (ECP) is suitable for attaining population inversion and 
offering the potential for laser emission in the spectral region between 12 and $53 \mathrm{~nm}$ from the Ge XXIII ion.

This class of lasers can be achieved under the suitable conditions of pumping power as well as electron density. If the positive gains obtained previously for some transitions in the ions under studies (Ge XXIII ion) together with the calculated parameters can be achieved experimentally, then successful low-cost electron collisional pumping XUV and soft X-ray lasers can be developed for various applications. The results have suggested the laser transitions in the Ge XXIII plasma ion (see Table 4), as the most promising laser emission lines in the XUV and soft X-ray spectral regions.

\section{References}

[1] Gudzenko, L.I. and Shelepin, L.A. (1966) Amplification of Radiation in the Decay of a Maximally Ionized Plasma. Journal of Applied Mechanics and Technical Physics, 7, 82.

[2] Maxon, S., Hagelstein, P., Reed, K. and Scofield, J. (1985) A Gas Puff Soft X-Ray Laser Target Design. Journal of Applied Physics, 57, 971. http://dx.doi.org/10.1063/1.334700

[3] Vinogradov, A.V., Sobelman, I. and Yukov, E. (1977) Population Inversion of Transitions in Neon-Like Ions. Soviet Journal of Quantum Electronics, 7, 32. http://dx.doi.org/10.1070/QE1977v007n01ABEH008807

[4] King, R.E., Pert, G.J., McCabe, S.P., Simms, P.A., MacPhee, A.G., Lewis, C.L.S., et al. (2001) Saturated X-Ray Lasers at 196 A and 73 A Pumped by Picosecond Travelling Wave Excitation. Physical Review A, 64, Article ID: 053810. http://dx.doi.org/10.1103/PhysRevA.64.053810

[5] Rocca, J.J., Shlyaptsev, V., Tomasel, F.G., Cortázar, O.D., Hartshorn, D. and Chilla, J.L.A. (1994) Demonstration of a Discharge Pumped Table Top Soft X-Ray Laser. Physical Review Letters, 73, 2192. http://dx.doi.org/10.1103/PhysRevLett.73.2192

[6] Tomsel, F.G., Rocca, J.J., Shlyaptsev, V.N. and Macchietto, C.D. (1997) Lasing at $60.8 \mathrm{~nm}$ in Ne-Like Sulfur Ions in Ablated Material Excited by a Capillary Discharge. Physical Review A, 55, 1437.

[7] Healy, S.P., Janulewicz, K.A. and Pert, G.J. (1997) Short Wavelength Lasing on Collisionally Pumped, Highly Excited 2s Hole States of Neon Like Ions in Performed Plasmas Irradiated with Picosecond Pulses. Optics Communications, 144, 24-30. http://dx.doi.org/10.1016/S0030-4018(97)00287-3

[8] Simms, P.A., McCabe, S. and Pert, G.J. (1998) Comparison of Neon Like Germanium Inner Shell and Nickel Like Gadolinium at $62 \mathrm{~A}$ and $66 \mathrm{~A}$ by Picosecond Heating of a Performed Plasma. Optics Communications, 153, $164-171$.

[9] Norton, B.A. and Peacock, N.J. (1975) Population Inversion in Laser-Produced Plasmas by Pumping with OpacityBroadened Lines. Journal of Physics B, 8, 989. http://dx.doi.org/10.1088/0022-3700/8/6/026

[10] Bhagavatula, V.A. (1976) Soft X-Ray Population Inversion by Resonant Photoexcitation in Multicomponent Laser Plasmas. Journal of Applied Physics, 47, 4535. http://dx.doi.org/10.1063/1.322425

[11] Nilsen, J., Beiersdorfer, P., Elliott, S.R., Phillips, T.W., Bryunetkin, B.A., Dyakin, V.M., Pikuz, T.A., Faenov, A.Ya., Pikuz, S.A., von Goeler, S., Bitter, M., Loboda, P.A., Lykov, V.A. and Politov, V.Yu. (1994) article title. Physical Review A, Article ID: 502143.

[12] Qi, N. and Krishnan, M. (1988) Enhancement of Ultraviolet Laser Plasma Emission Produced in a Strong Static Electric Field. Optics Communications, 67, 124-128.

[13] Feldman, U., Bhatia, A.K. and Suckewer, S. (1983) Short Wavelength Laser Calculations for Electron Pumping in Neon-Like Krypton (Kr XXVII). Journal of Applied Physics, 54, 2188. http://dx.doi.org/10.1063/1.332371

[14] Feldman, U., Seely, J.F. and Bhatia, A.K. (1984) Scaling of Collisionally Pumped 3s-3p Lasers in the Neon Isoelectronic Sequence. Journal of Applied Physics, 56, 2475. http://dx.doi.org/10.1063/1.334308

[15] Chapline, G. and Wood, L. (1975) X-Ray Lasers. Physics Today, 28, 40. http://dx.doi.org/10.1063/1.3069004

[16] Vinogradov, A.V. and Shlyaptsev, V.N. (1980) Calculations of Population Inversion Due to Transitions in Multiply Charged Neon-Like Ions in the 200-2000 Å Range. Soviet Journal of Quantum Electronics, 10, 754. http://dx.doi.org/10.1070/QE1980v010n06ABEH010287

[17] Goldstein, W.H., Oreg, J., Zigler, A., Bar-Shalom, A. and Klapisch, M. (1988) Gain Predictions for Nickel-Like Gadolinium from a 181-Level Multiconfigurational Distorted-Wave Collisional-Radiative Model. Physical Review A, 38, 1797. http://dx.doi.org/10.1103/PhysRevA.38.1797

[18] Sobel'man, I.I. (1979) Introduction to the Theory of Atomic Spectra, International Series of Monographs in Natural Philosophy. Vol. 40, Pergamon Press, Oxford.

[19] Hagelstein, P.L. (1986) Relativistic Distorted-Wave Results for Nickel-Like Gadolinium. Physical Review A, $34,874$. http://dx.doi.org/10.1103/PhysRevA.34.874

[20] Zhang, H.L., Samson, D.H. and Fontes, C.J. (1991) Relativistic Distorted-Wave Collision Strengths and Oscillator 
Strengths for the $33 \mathrm{Ni-Like} \mathrm{Ions} \mathrm{with} 60 \leq \mathrm{Z} \leq 92$. Atomic Data and Nuclear Data Tables, 48, 91-163. http://dx.doi.org/10.1016/0092-640X(91)90024-X

[21] Feldman, U., Doschek, G.A., Seely, J.F. and Bhatia, A.K. (1985) Short Wavelength Laser Calculations for Electron Pumping in Be I and B I Isoelectronic Sequences (18 $\leq \mathrm{Z} \leq 36)$. Journal of Applied Physics, 58, 2909. http://dx.doi.org/10.1063/1.335838

[22] Hibbert, A., Ledourneuf, M. and Mohan, M. (1993) Energies, Oscillators Strengths, and Life Times for Neon-Like Ions up to Kr XXVII. Atomic Data and Nuclear Data Tables, 53, 23-112.

[23] Daido, H., Kodama, R., Murai, K., Yuan, G., Takagi, M., Kato, Y., Choi, I.W. and Nam, C.H. (1995) Significant Improvement in the Efficiency and Brightness of the $J=0-1$ 19.6-nm Line of the Germanium Laser by Use of Double-Pulse Pumping. Optics Letters, 20, 61-63.

[24] NIST. http://F:/NIST/NIST\%20ASD\%20Levels\%20Output32.htm 
Scientific Research Publishing (SCIRP) is one of the largest Open Access journal publishers. It is currently publishing more than 200 open access, online, peer-reviewed journals covering a wide range of academic disciplines. SCIRP serves the worldwide academic communities and contributes to the progress and application of science with its publication.

Other selected journals from SCIRP are listed as below. Submit your manuscript to us via either submit@scirp.org or Online Submission Portal.
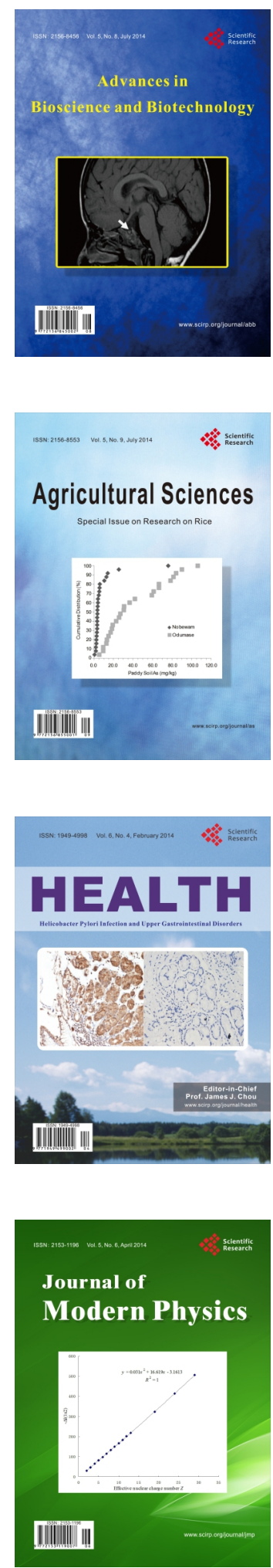
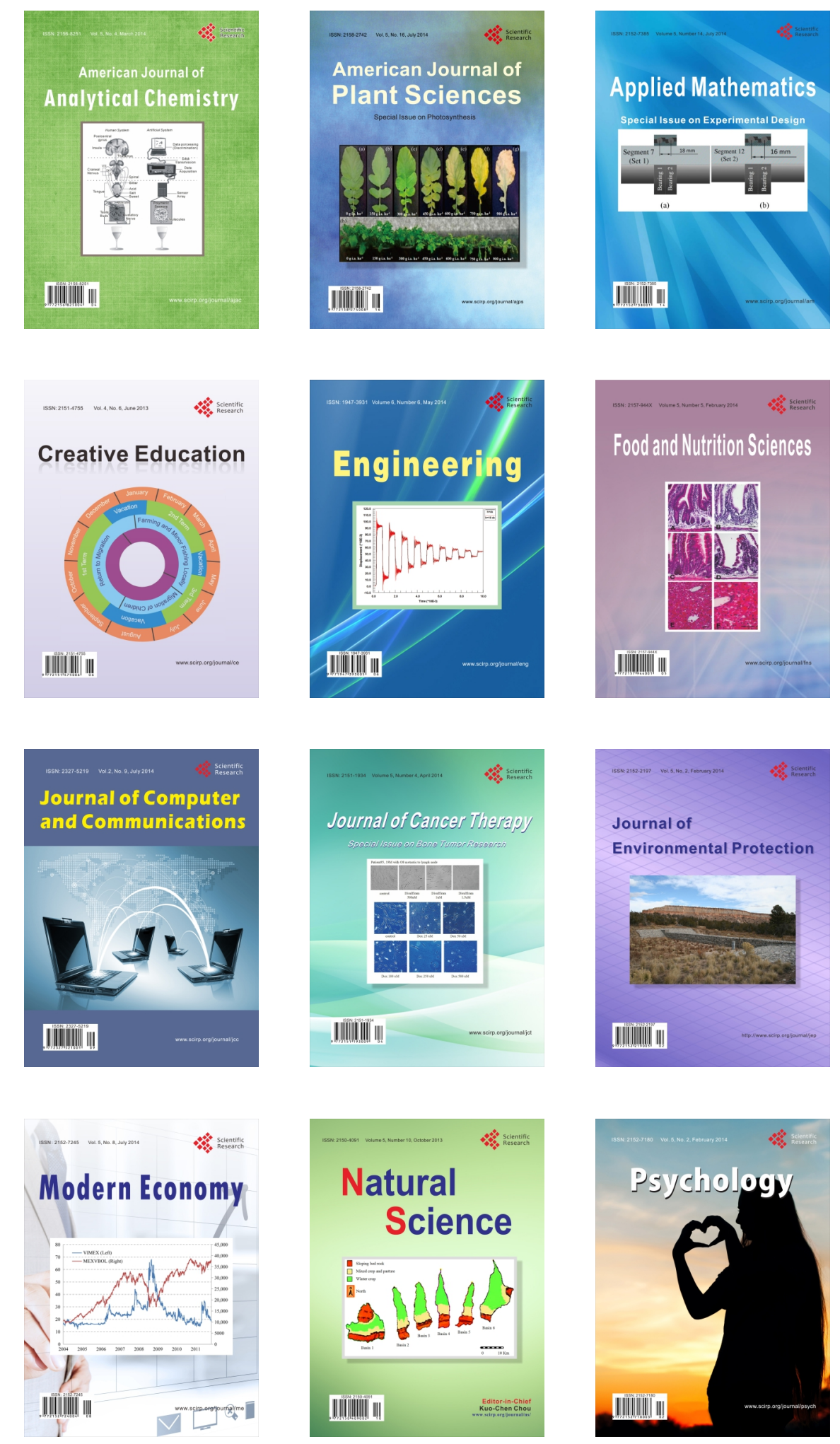